\title{
Maximum Likelihood Estimation of State Space Models From Frequency Domain Data
}

\author{
Adrian Wills, Brett Ninness, and Stuart Gibson
}

\begin{abstract}
This paper addresses the problem of estimating linear time invariant models from observed frequency domain data. Here an emphasis is placed on deriving numerically robust and efficient methods that can reliably deal with high order models over wide bandwidths. This involves a novel application of the expectation-maximization algorithm in order to find maximum likelihood estimates of state space structures. An empirical study using both simulated and real measurement data is presented to illustrate the efficacy of the solutions derived here.
\end{abstract}

Index Terms-Expectation-maximization (EM), maximumlikelihood (ML).

\section{INTRODUCTION}

A widely used approach to the problem of linear dynamic system identification is to first obtain measurements of a system's frequency response, and then take as estimate the model of given order whose response most closely matches these measurements [16], [18]. This technique may be employed because the system observations naturally present themselves in the frequency domain, such as in the area of vibration analysis [11]. Alternatively, it may be used in order to obtain some of the natural advantages that flow from a frequency domain approach, such as ease of combining separate data sets, ability to compress large (time domain) data records, and ease of accommodating continuous time models [21].

However, the approach is not without difficulties. In most cases finding the estimate involves solving a non-convex nonlinearly parametrized optimization problem. This necessitates some sort of iterative search such as a gradient based technique [5]. Furthermore, when the observed data is in the frequency domain, it can have a very wide dynamic range involving several orders of magnitude, with features at small magnitudes being as significant from a modelling viewpoint as features at large magnitudes. Finally, it may be required for the estimated model to fit the observed data over several decades of frequency.

The combined effect of these issues is that solving a frequency domain estimation problem often implies solving a complex optimization problem subject to significant numerical conditioning difficulties. A wide variety of techniques have been

Manuscript received March 13, 2007; revised January 21, 2007. Current version published January 14, 2009. This work was supported by the Australian Research Council. Recommended by Associate Editor H. Hjalmarsson.

A. Wills and B. Ninness are with the School of Electrical Engineering and Computer Science, University of Newcastle, Newcastle NSW 2300, Australia (e-mail: adrian.wills@newcastle.edu.au; brett.ninness@ @ewcastle.edu.au).

S. Gibson is with Nomura, London EC1A 4NP, U.K. (e-mail: stuart.h. gibson@gmail.com).

Color versions of one or more of the figures in this paper are available online at http://ieeexplore.ieee.org.

Digital Object Identifier 10.1109/TAC.2008.2009485 developed to cope with these difficulties, with a commonly used approach being one of Gauss-Newton type search with respect to models which are specifically parametrized to ameliorate numerical conditioning issues [21].

This paper explores a new approach of tackling the model fitting problem via the expectation-maximization (EM) algorithm. As opposed to gradient-based search which applies to the general class of smooth cost functions, the EM method was specifically developed to tackle (possibly non-smooth) maximum-likelihood (ML) criteria. It was originally developed in the statistics literature [4], and enjoys a reputation in that field of providing a particularly robust means for iteratively computing ML estimates.

However, it has not (to the authors knowledge) previously been applied to the frequency domain estimation problems studied here, although the current authors have studied the method for both linear and non-linear estimation from time domain data in [6], [7]. In that context, it was found to provide a method that was particularly robust against capture in local minima. It was also observed to enjoy favourable numerical conditioning and reasonable computational load which scaled only modestly with increasing model complexity.

These advantages in the time domain case motivated the frequency domain study here, and as will be illustrated, the same benefits apply in this new setting. Like gradient based search, the algorithm involved is still iterative in nature and consists of two key steps. In the first, a smoothed state estimate based on the current iterate model parameters is computed. In the second, this state estimate is used to update the model parameters via solving two maximization problems. Unlike the time domain cases studied in [6], [7], this maximization step cannot be found in closed form and a gradient based search is required. However, the problems involved are unimodal (i.e., quasi-convex-see [1]), and hence simply solved.

The efficacy of the method is illustrated here empirically on a range of simulation studies of varying system dimension, and on two real world problems involving a cantilevered beam and a vibrating mechanical system. For readers wishing to perform their own evaluation, MATLAB-based software implementing the methods derived and studied here is freely available at sigpromu.org/idtoolbox.

The paper is organized as follows. In Section II we define the model structure and noise assumptions used in the remainder of the paper. Section III reviews the ML method for state-space models in the frequency-domain. This leads to the description of the EM algorithm in Section IV. Section V detailing the expectation step and Sections VI-VII detailing the maximization together with computational considerations may be skipped by readers interested only in the resulting algorithm, which is summarized in Section VIII. Its convergence properties are analysed 
in Section IX. The EM method is profiled against other competitive methods for MIMO state-space models in Section X, and Section XII concludes the paper.

\section{Model Structure AND Available Data}

This paper assumes the availability of a set of measurements $\left\{Y\left(\omega_{k}\right) \triangleq Y_{k}\right\}_{k=1}^{N}, Y_{k} \in \mathbf{C}^{p \times m}$ of a system's frequency response at a set $\left\{\omega_{k}\right\}_{k=1}^{N}$ of frequencies that need not be equally spaced. Measurements $\left\{U\left(\omega_{k}\right) \triangleq U_{k}\right\}_{k=1}^{N}, U_{k} \in \mathbf{C}^{m \times m}$ of the input spectrum at these same frequencies are also assumed available. By allowing $p>1, m>1$ the cases of multiple output, multiple input, or both are all catered for. More specifically, the $i, \ell$ th element of the matrix $Y_{k}$ is the response at the $i$ th output on the $\ell$ th excitation experiment, and the $i, \ell$ th element of $U_{k}$ is the excitation on the $i$ th input during the $\ell$ th experiment [21, Section 2.7].

The topic of this paper is to address the problem of finding matrices $A, B, C, D$ in a state space model such that the associated frequency response

$$
G\left(\gamma_{k}\right)=C\left(\gamma_{k} I-A\right)^{-1} B U_{k}+D ; \quad \gamma_{k}=e^{j \omega_{k}} \text { or } j \omega_{k}
$$

matches that of an underlying true linear system producing the measurements $\left\{Y_{k}\right\}$ in response to $\left\{U_{k}\right\}$ as closely as possible. Via the choice of $\gamma_{k}$ in (1) both discrete time and continuous time models are accommodated. Furthermore, the common situation of Frequency-Response-Function (FRF) data is addressed by setting $U_{k}=I_{m}$, where $I_{m}$ is the $m \times m$ identity matrix.

To address this estimation problem, the following state space model structure is employed:

$$
\begin{aligned}
{\left[\begin{array}{c}
\gamma_{k} X_{k} \\
Y_{k}
\end{array}\right] } & =\left[\begin{array}{ll}
A & B \\
C & D
\end{array}\right]\left[\begin{array}{l}
X_{k} \\
U_{k}
\end{array}\right]+\left[\begin{array}{c}
W_{k} \\
E_{k}
\end{array}\right] \\
\gamma_{k} & =e^{j \omega_{k}} \text { or } j \omega_{k} .
\end{aligned}
$$

Here, $A \in \mathbf{R}^{n \times n}, B \in \mathbf{R}^{n \times m}, C \in R^{p \times n}$ and $D \in \mathbf{R}^{p \times m}$ are the system matrices to be estimated. The element $X_{k} \in \mathbf{C}^{n \times m}$ is the frequency response of the system state in response to the excitation $U_{k}=U\left(\omega_{k}\right)$. The terms $W_{k} \in \mathbf{C}^{n \times m}$ and $E_{k} \in$ $\mathbf{C}^{p \times m}$ model corruptions in the frequency response measurements. There are assumed to be zero mean i.i.d. random variables that are circular complex Normally distributed according to

$$
\left[\begin{array}{c}
\operatorname{vec}\left\{W_{k}\right\} \\
\operatorname{vec}\left\{E_{k}\right\}
\end{array}\right] \sim \mathcal{N}_{c}\left(\left[\begin{array}{l}
\mathbf{0} \\
\mathbf{0}
\end{array}\right],\left[\begin{array}{cc}
I_{m} \otimes Q & \mathbf{0} \\
\mathbf{0} & I_{m} \otimes R
\end{array}\right]\right) .
$$

Here vec $\{X\} \in \mathbf{C}^{n m}$ is the vector obtained by stacking the left to right columns of the matrix $X \in \mathbf{C}^{n \times m}$ on top of one another, and $\otimes$ is the Kronecker product.

The circular complex Normal distribution is defined such that if $Z \sim \mathcal{N}_{c}(\mu, P)$ for a vector $Z \in \mathbf{C}^{n}$ then its real and imaginary parts are distributed as

$$
\left[\begin{array}{l}
\operatorname{Re}\{Z\} \\
\operatorname{Im}\{Z\}
\end{array}\right] \sim \mathcal{N}\left(\left[\begin{array}{l}
\operatorname{Re}\{\mu\} \\
\operatorname{Im}\{\mu\}
\end{array}\right], \frac{1}{2}\left[\begin{array}{cc}
\operatorname{Re}\{P\} & -\operatorname{Im}\{P\} \\
\operatorname{Im}\{P\} & \operatorname{Re}\{P\}
\end{array}\right]\right)
$$

where $\mathcal{N}$ denotes a standard Gaussian density, and $Z$ has probability density function

$$
p(Z)=\frac{1}{\pi^{n}|P|} \exp \left(-(Z-\mu)^{\star} P^{-1}(Z-\mu)\right)
$$

with $(\cdot)^{\star}$ denoting the complex conjugate transpose of $(\cdot)$.

This assumption of i.i.d. circular complex Normal distribution is widely employed in the frequency domain estimation literature [21], [16], [17]. It is motivated by several considerations. The most significant being that the discrete Fourier transform (DFT) of a white Gaussian time domain measurement corruption has an i.i.d. circular complex Normal distribution, and that the DFT of a stationary bounded variance time domain measurement converges in distribution as the time domain measurement length grows to a sequence of i.i.d. circular complex Normal random variables [2]. Coupling these stochastic assumptions with state space models has also been previously considered in [16], [17] where the corresponding 'innovations form' of (2) is employed.

Finally, it is important to emphasize that the model (2) does not explicitly account for any non-zero initial conditions affecting the frequency domain measurements $\left\{Y_{k}\right\},\left\{U_{k}\right\}$. These typically decrease in size with increasing time domain data length [16], and it is assumed that this length is sufficient that they are negligible.

\section{MAXIMUM LiKeliHOOD ESTIMATION}

Given the model structure (2), (3), the work here addresses the problem of using the data $\left\{U_{k}\right\}_{k=1}^{N}$ and $\left\{Y_{k}\right\}_{k=1}^{N}$ to form an estimate $\hat{\theta}$ of the system parametrization

$$
\theta \triangleq\left[\beta^{T}, \eta^{T}, \operatorname{vec}\{Q\}^{T}, \operatorname{vec}\{R\}^{T}\right]^{T}
$$

where

$$
\begin{gathered}
\beta \triangleq \operatorname{vec}\{\Delta\}, \quad \Delta \triangleq[A, B], \\
\eta \triangleq \operatorname{vec}\{\Gamma\}, \quad \Gamma \triangleq[C, D] .
\end{gathered}
$$

It is worth pointing out that $\beta$ and $\Delta$ are isomorphic, and likewise $\eta$ and $\Gamma$ are isomorphic; we exploit this one-to-one correspondence throughout the remainder of the paper. The parameter vector $\theta$ is constrained to the parameter set $\Theta$ defined via

$$
\Theta=\Theta_{1} \times \Theta_{2} \times \Theta_{3}, \quad[\beta, \eta] \in \Theta_{1}, \quad Q \in \Theta_{2}, \quad R \in \Theta_{3}
$$

where $\Theta_{1}$ is a closed hypercube in $\mathbf{R}^{\ell}, \ell=n^{2}+n m+n p+p^{2}$, and $\Theta_{2}$ is a closed and bounded subset of $\mathbf{R}^{n \times n}$ for which all $Q \in \Theta_{2}$ are symmetric and positive definite. Similarly, $\Theta_{3}$ is a closed and bounded subset of $\mathbf{R}^{p \times p}$ for which all $R \in \Theta_{3}$ are symmetric and positive definite.

For estimation purposes, this paper employs the well-known maximum likelihood criterion

$$
\widehat{\theta} \triangleq \underset{\theta \in \Theta}{\arg \max } p_{\theta}\left(Y_{1}, \ldots, Y_{N}\right)
$$

which has well known properties of statistical optimality [21]. This approach requires computation of the likelihood $p_{\theta}\left(Y_{1}, \ldots, Y_{M}\right)$. To achieve this, note that via (2)

$$
Y_{k}=G_{k} U_{k}+T_{k} W_{k}+E_{k}
$$

where $W_{k} \triangleq W\left(\omega_{k}\right), E_{k} \triangleq E\left(\omega_{k}\right)$ and

$$
T_{k} \triangleq C\left(\gamma_{k} I-A\right)^{-1}, \quad G_{k} \triangleq T_{k} B+D
$$


so that

$$
\operatorname{vec}\left\{Y_{k}\right\} \sim \mathcal{N}_{c}\left(\operatorname{vec}\left\{G_{k} U_{k}\right\}, I_{m} \otimes P_{k}\right)
$$

where

$$
P_{k} \triangleq C A_{k}^{-1} Q A_{k}^{-\star} C^{T}+R, \quad A_{k} \triangleq \gamma_{k} I-A .
$$

Note that this requires that $A$ have no eigenvalues on the stability boundary, which is assumed true in the remainder of the paper. To continue, by the independence assumptions of $W_{k}$ and $W_{\ell}$ for $k \neq \ell$, and similarly for $E_{k}, E_{\ell}$

$$
\begin{aligned}
& p_{\theta}\left(Y_{1}, \ldots, Y_{N}\right)=\prod_{k=1}^{N} p_{\theta}\left(Y_{k}\right)=\prod_{k=1}^{N} \frac{1}{\pi^{n}\left|P_{k}\right|^{m}} \\
& \cdot \exp \left\{-\operatorname{Tr}\left\{\left(Y_{k}-G_{k} U_{k}\right)^{\star} P_{k}^{-1}\left(Y_{k}-G_{k} U_{k}\right)\right\}\right\} .
\end{aligned}
$$

Actually, since $\log x$ is monotonic in $x$, then the maximum likelihood estimate can be equivalently defined as

$$
\widehat{\theta}=\underset{\theta \in \Theta}{\arg \max } L(\theta)
$$

where $L(\theta)$ is the log-likelihood function

$$
L(\theta)=\log p_{\theta}\left(Y_{1}, \ldots, Y_{N}\right) .
$$

In this frequency domain case, (13) makes it clear that (after dropping terms independent of $\theta$ )

$$
\begin{aligned}
& L(\theta)=-m \\
& \cdot \sum_{k=1}^{N} \log \left|P_{k}\right|-\operatorname{Tr}\left\{\left(Y_{k}-G_{k} U_{k}\right)^{\star} P_{k}^{-1}\left(Y_{k}-G_{k} U_{k}\right)\right\} .
\end{aligned}
$$

The essential difficulty now is that via the formulation of $G_{k}$, $T_{k}$ and $P_{k}, L(\theta)$ is parametrized in quite a complicated way by $\theta$. As such, the computation of $\widehat{\theta}$ via minimization of $L(\theta)$ is not straightforward, and certainly it requires some sort of iterative search procedure to be used.

Previous work has addressed this via the employment of a gradient-based search method such as Gauss-Newton iteration or a variant thereof [15], [21]. However, this previous work does not specifically consider MIMO state-space model structures with state noise. This is possibly due to the inherent difficulty of calculating the gradient and Hessian of the ML cost function (31). The work here avoids this difficulty by employing the so-called EM algorithm.

\section{EM ALGORITHM}

To explain and motivate the EM Algorithm it will be convenient to define the full output and full state information sequences as

$$
\bar{Y} \triangleq\left\{Y_{1}, \ldots, Y_{N}\right\}, \quad \bar{X} \triangleq\left\{X_{1}, \ldots, X_{N}\right\} .
$$

An essential ingredient in the understanding of the EM Algorithm is the recognition that via Bayes' rule $p(\bar{Y})=$
$p(\bar{X}, \bar{Y}) / p(\bar{X} \mid \bar{Y})$ and hence the log likelihood $L(\theta)$ in (15) can be decomposed as

$$
L(\theta)=\log p_{\theta}(\bar{Y})=\log p_{\theta}(\bar{X}, \bar{Y})-\log p_{\theta}(\bar{X} \mid \bar{Y}) .
$$

Note that in general, the component $\bar{X}$ is termed the "missing data" and its choice is the essential design variable in the deployment of the EM method [4], [6], [7]. Its selection here as the states in an underlying state-space model is part of the adaptation of the EM approach to this specific frequency domain estimation setting.

A second essential point is that the expectation of $L(\theta)$ given the output measurements $\bar{Y}$ and with respect to a probability density function implied by parameters $\theta^{\prime}$ leaves $L(\theta)$ unchanged. That is

$$
\mathrm{E}_{\theta^{\prime}}\{L(\theta) \mid \bar{Y}\}=\int \log p_{\theta}(y) p_{\theta^{\prime}}(y \mid y=\bar{Y}) \mathrm{d} y=\log p_{\theta}(\bar{Y})
$$

since clearly $p_{\theta^{\prime}}(y \mid y=\bar{Y})=\delta(y-\bar{Y})$ a Dirac delta. Therefore, via (18)

$$
L(\theta)=\mathcal{Q}\left(\theta, \theta^{\prime}\right)-\mathcal{V}\left(\theta, \theta^{\prime}\right) .
$$

where

$$
\begin{aligned}
& \mathcal{Q}\left(\theta, \theta^{\prime}\right) \triangleq \mathrm{E}_{\theta^{\prime}}\left\{\log p_{\theta}(\bar{X}, \bar{Y}) \mid \bar{Y}\right\} \\
& \mathcal{V}\left(\theta, \theta^{\prime}\right) \triangleq \mathrm{E}_{\theta^{\prime}}\left\{\log p_{\theta}(\bar{X} \mid \bar{Y}) \mid \bar{Y}\right\} .
\end{aligned}
$$

The final key component is that via (19)

$$
L(\theta)-L\left(\theta^{\prime}\right)=\mathcal{Q}\left(\theta, \theta^{\prime}\right)-\mathcal{Q}\left(\theta^{\prime}, \theta^{\prime}\right)+\underbrace{\mathcal{V}\left(\theta^{\prime}, \theta^{\prime}\right)-\mathcal{V}\left(\theta, \theta^{\prime}\right)}_{\geq 0}
$$

where the indicated positivity arises since the term involved is easily established via the definition in (19) as the Kullback-Leibler divergence metric between $p_{\theta}(\bar{X} \mid \bar{Y})$ and $p_{\theta^{\prime}}(\bar{X} \mid \bar{Y})[3]$.

It follows immediately that if $\mathcal{Q}\left(\theta, \theta^{\prime}\right)>\mathcal{Q}\left(\theta^{\prime}, \theta^{\prime}\right)$ then $L(\theta)>L\left(\theta^{\prime}\right)$ which naturally suggests what is known as the EM algorithm which takes an approximation $\widehat{\theta}_{i}$ of the ML estimate $\widehat{\theta}$ given by (14) and updates it to an expected better one $\hat{\theta}_{i+1}$ according to:

1) $\boldsymbol{E}$ Step

$$
\text { Calculate : } \quad \mathcal{Q}\left(\theta, \widehat{\theta}_{i}\right)
$$

\section{2) M Step}

$$
\text { Compute : } \quad \widehat{\theta}_{i+1}=\underset{\theta \in \Theta}{\arg \max } \mathcal{Q}\left(\theta, \widehat{\theta}_{i}\right) .
$$

Like gradient based search, a single iteration is unlikely to find the optimizer $\widehat{\theta}$ and hence the above steps are iterated multiple times to produce a sequence $\left\{\widehat{\theta}_{0}, \widehat{\theta}_{1}, \widehat{\theta}_{2}, \ldots\right\}$ of increasingly good approximations to $\widehat{\theta}$. The iterations are usually terminated using a standard criterion such as the relative decrease of $L(\theta)$ falling below a pre-defined threshold [5]. 


\section{THE E-STEP}

To implement the EM Algorithm for frequency domain estimation using the missing data choice $\bar{X}$ given by (17) it is clearly necessary to derive a method for computing $\mathcal{Q}\left(\theta, \widehat{\theta}_{i}\right)$. This may be simply achieved via the results of the following two Lemmas.

Lemma 1: The function $\mathcal{Q}\left(\theta, \widehat{\theta}_{i}\right)$ defined via (19) may be expressed as [recall the definitions in (7) and (8)]

where

$$
\mathcal{Q}\left(\theta, \widehat{\theta}_{i}\right)=-\mathcal{Q}_{\Gamma}\left(\eta, R, \widehat{\theta}_{i}\right)-\mathcal{Q}_{\Delta}\left(\beta, Q, \widehat{\theta}_{i}\right)
$$

$$
\begin{aligned}
\mathcal{Q}_{\Gamma}\left(\eta, R, \widehat{\theta}_{i}\right) \triangleq & m N \log |R| \\
& +\operatorname{Tr}\left\{R^{-1}\left[\Phi-\Gamma \Psi^{\star}-\Psi \Gamma^{T}+\Gamma \Sigma \Gamma^{T}\right]\right\} \\
\mathcal{Q}_{\Delta}\left(\beta, Q, \widehat{\theta}_{i}\right) \triangleq & m N \log |Q|-2 m \sum_{k=1}^{N} \operatorname{Re}\left\{\log \left|A_{k}\right|\right\} \\
& +\operatorname{Tr}\left\{Q^{-1}\left[\Lambda-\Delta \Omega^{\star}-\Omega \Delta^{T}+\Delta \Sigma \Delta^{T}\right]\right\}
\end{aligned}
$$

where $A_{k}$ has been defined in (12) and

$$
\begin{aligned}
& \Phi \triangleq \sum_{k=1}^{N} Y_{k} Y_{k}^{\star}, \Omega \triangleq \sum_{k=1}^{N} \mathrm{E}_{\widehat{\theta}_{i}}\left\{\gamma_{k} X_{k} Z_{k}^{\star} \mid Y_{k}\right\} \\
& \Psi \triangleq \sum_{k=1}^{N} \mathrm{E}_{\widehat{\theta}_{i}}\left\{Y_{k} Z_{k}^{\star} \mid Y_{k}\right\}, \quad \Sigma \triangleq \sum_{k=1}^{N} \mathrm{E}_{\widehat{\theta}_{i}}\left\{Z_{k} Z_{k}^{\star} \mid Y_{k}\right\} \\
& \Lambda \triangleq \sum_{k=1}^{N} \mathrm{E}_{\widehat{\theta}_{i}}\left\{\gamma_{k}^{\star} \gamma_{k} X_{k} X_{k}^{\star} \mid Y_{k}\right\}, Z_{k} \triangleq\left[\begin{array}{c}
X_{k} \\
U_{k}
\end{array}\right]
\end{aligned}
$$

Proof: See Appendix I.

This reduces the problem of computing $\mathcal{Q}\left(\theta, \widehat{\theta}_{i}\right)$ to one of computing a smoothed state estimate and its covariance, which itself may be achieved by the results of the following Lemma.

Lemma 2: Assume that observed data $Y_{k}$ obeys the model (2), which is parametrized by a given vector $\theta$ according to (6). Then

$$
\begin{aligned}
\widehat{X}_{k} \triangleq & \mathrm{E}_{\theta}\left\{X_{k} \mid Y_{k}\right\}=A_{k}^{-1} B U_{k} \\
& +S_{k} R_{k}^{-1}\left(Y_{k}-C A_{k}^{-1} B U_{k}-D U_{k}\right),
\end{aligned}
$$

and $\mathrm{E}_{\theta}\left\{X_{k} X_{k}^{\star} \mid Y_{k}\right\}=\widehat{X}_{k} \widehat{X}_{k}^{\star}+Q_{k}-S_{k} R_{k}^{-1} S_{k}^{\star}$

where $A_{k}$ has been defined in (12) and

$$
Q_{k} \triangleq A_{k}^{-1} Q A_{k}^{-\star}, \quad S_{k} \triangleq Q_{k} C^{T}, \quad R_{k} \triangleq C Q_{k} C^{T}+R .
$$

Proof: According to the model (8)

$$
\left[\begin{array}{c}
X_{k} \\
Y_{k}
\end{array}\right] \sim \mathcal{N}_{c}\left\{\left[\begin{array}{c}
A_{k}^{-1} B U_{k} \\
C A_{k}^{-1} B U_{k}+D U_{k}
\end{array}\right],\left[\begin{array}{cc}
Q_{k} & S_{k} \\
S_{k}^{\star} & R_{k}
\end{array}\right]\right\}
$$

Therefore, by Lemma 12 in Appendix V

$$
\left(X_{k} \mid Y_{k}\right) \sim \mathcal{N}_{c}\left(\mu_{k}, \Pi_{k}\right)
$$

where

$$
\begin{aligned}
\mu_{k} & =A_{k}^{-1} B U_{k}+S_{k} R_{k}^{-1}\left(Y_{k}-C A_{k}^{-1} B U_{k}-D U_{k}\right) \\
\Pi_{k} & =Q_{k}-S_{k} R_{k}^{-1} S_{k}^{\star} .
\end{aligned}
$$

\section{THE M-STEP}

Together, Lemmas 1 and 2 provide a simple means for executing the 'expectation step' (23) of computing $\mathcal{Q}\left(\theta, \widehat{\theta}_{i}\right)$. This leads to consideration of the 'maximization step' (24). Unfortunately, this is not so straightforward since it can only partially be achieved by closed form expressions.

Recall that via (25), $\mathcal{Q}\left(\theta, \widehat{\theta}_{i}\right)$ can be decomposed into two components $\mathcal{Q}_{\Delta}\left(\beta, Q, \widehat{\theta}_{i}\right)$ and $\mathcal{Q}_{\Gamma}\left(\eta, R, \widehat{\theta}_{i}\right)$ which depend in a non-coupled fashion on particular elements of the state space description $A, B, Q$ and $C, D, R$, respectively, as defined via the parametrization in (7), (8).

This allows the optimization problem (24) to be decomposed into smaller ones. The first of these involves maximization (minimization of $\mathcal{Q}_{\Gamma}\left(\eta, R, \widehat{\theta}_{i}\right)$ and $\left.\mathcal{Q}_{\Delta}\left(\beta, Q, \widehat{\theta}_{i}\right)\right)$ with respect to the noise parametrizations $R$ and $Q$. This subproblem does have a closed form solution.

Lemma 3: For any $\eta=\operatorname{vec}\{[C, D]\}$

$$
\widehat{R}(\eta) \triangleq \frac{1}{m N}\left[\Phi-\Gamma \Psi^{\star}-\Psi \Gamma^{T}+\Gamma \Sigma \Gamma^{T}\right]
$$

is a stationary point of $\mathcal{Q}_{\Gamma}(\eta, R)$ with respect to $R$ and

$$
\left.\frac{1}{m N} \frac{\partial^{2} \mathcal{Q}_{\Gamma}(\eta, R)}{\partial \operatorname{vec}\{R\} \partial \operatorname{vec}\{R\}^{T}}\right|_{R=\widehat{R}(\eta)}=\widehat{R}(\eta) \otimes \widehat{R}(\eta) .
$$

Likewise, for any $\beta=\operatorname{vec}\{[A, B]\}$

$$
\widehat{Q}(\beta) \triangleq \frac{1}{m N}\left[\Lambda-\Delta \Omega^{\star}-\Omega \Delta^{T}+\Delta \Sigma \Delta^{T}\right]
$$

is a stationary point of $\mathcal{Q}_{\Delta}(\beta, Q)$ with respect to $Q$ and

$$
\left.\frac{1}{m N} \frac{\partial^{2} \mathcal{Q}_{\Delta}(\beta, Q)}{\partial \operatorname{vec}\{Q\} \partial \operatorname{vec}\{Q\}^{T}}\right|_{Q=\widehat{Q}(\beta)}=\widehat{Q}(\beta) \otimes \widehat{Q}(\beta)
$$

Proof: By Lemma 13

$$
\begin{aligned}
& \frac{\partial}{\partial R} \mathcal{Q}_{\Gamma}(\eta, R) \\
& \quad=m N R^{-1}-R^{-1}\left[\Phi-\Gamma \Psi^{\star}-\Psi \Gamma^{T}+\Gamma \Sigma \Gamma^{T}\right] R^{-1}
\end{aligned}
$$

which is clearly equal to zero for $R=\widehat{R}(\eta)$ given by (33). A similar argument holds for $\widehat{Q}(\beta)$. Furthermore

$$
\frac{\partial \mathcal{Q}_{\Gamma}(\eta, R)}{\partial \operatorname{vec}\{R\}}=m N \operatorname{vec}\left\{R^{-1}-R^{-1} \widehat{R}(\eta) R^{-1}\right\} \text {. }
$$

Lemma 13 and the product rule then yields

$$
\begin{aligned}
\frac{1}{m N} & \frac{\partial^{2} \mathcal{Q}_{\Gamma}(\eta, R)}{\partial \operatorname{vec}\{R\} \partial \operatorname{vec}\{R\}^{T}} \\
= & \frac{\partial \operatorname{vec}\left\{R^{-1}\right\}}{\partial \operatorname{vec}\{R\}^{T}}-\left[\left(\widehat{R}(\eta) R^{-1}\right)^{T} \otimes I\right] \frac{\partial \operatorname{vec}\left\{R^{-1}\right\}}{\partial \operatorname{vec}\{R\}^{T}} \\
& -\left[I \otimes\left(R^{-1} \widehat{R}(\eta)\right)\right] \frac{\partial \operatorname{vec}\left\{R^{-1}\right\}}{\partial \operatorname{vec}\{R\}^{T}} \\
= & {\left[\left(\widehat{R}(\eta) R^{-1}\right)^{T} \otimes I\right]\left(R^{-1} \otimes R^{-1}\right) } \\
& +\left[I \otimes\left(R^{-1} \widehat{R}(\eta)\right)\right]\left(R^{-1} \otimes R^{-1}\right)-R^{-1} \otimes R^{-1}
\end{aligned}
$$


Evaluating this at $R=\widehat{R}(\eta)$ then establishes (34), with (36) being obtained in an identical manner.

Therefore, provided the stationary points $\widehat{R}(\eta), \widehat{Q}(\beta)$ given by (33), (35) are positive definite, then they provide closed form expressions for local maximizers of $\mathcal{Q}\left(\theta, \widehat{\theta}_{i}\right)$ with respect to $R$ and $Q$.

Substituting them for the $R$ and $Q$ terms of $\mathcal{Q}_{\Gamma}\left(\eta, R, \widehat{\theta}_{i}\right)$ and $\mathcal{Q}_{\Delta}\left(\beta, Q, \widehat{\theta}_{i}\right)$ then leads to 'concentrated' versions that depend only on the system parameters $\beta=\operatorname{vec}\{\Delta\}=\operatorname{vec}\{[A, B]\}$ and $\eta=\operatorname{vec}\{\Gamma\}=\operatorname{vec}\{[C, D]\}$ according to

$$
\begin{aligned}
& \mathcal{Q}_{\Gamma}(\eta, \widehat{R}(\eta)) \\
& =m N \log \left|\frac{1}{m N}\left[\Phi-\Gamma \Psi^{\star}-\Psi \Gamma^{T}+\Gamma \Sigma \Gamma^{T}\right]\right|+m p N
\end{aligned}
$$

and

$$
\begin{array}{r}
\mathcal{Q}_{\Delta}(\beta, \widehat{Q}(\beta))=m N \log \left|\frac{1}{m N}\left[\Lambda-\Delta \Omega^{\star}-\Omega \Delta^{T}+\Delta \Sigma \Delta^{T}\right]\right| \\
+m n N-2 m \sum_{k=1}^{N} \operatorname{Re}\left\{\log \left|A_{k}\right|\right\} .
\end{array}
$$

Considering (39), since

$$
\begin{aligned}
& \Phi-\Gamma \Psi^{\star}-\Psi \Gamma^{T}+\Gamma \Sigma \Gamma^{T} \\
& \quad=\left(\Gamma-\Psi \Sigma^{-1}\right) \Sigma\left(\Gamma-\Psi \Sigma^{-1}\right)^{\star}+\Phi-\Psi \Sigma^{-1} \Psi^{\star}
\end{aligned}
$$

then it is tempting to conclude via Minkowski's inequality for determinants of positive definite matrices [9] that

$$
\widehat{\eta}=\operatorname{vec}\{\widehat{\Gamma}\}, \quad \widehat{\Gamma}=\Psi \Sigma^{-1}
$$

globally minimizes (39). Unfortunately, this overlooks the constraint that $\widehat{\eta}$ must be real valued, and it is not clear how it can be simply accommodated while retaining a closed form solution. As well, (40) has an additional complicating factor due to the presence of the $\sum_{k=1}^{N} \operatorname{Re}\left\{\log \left|A_{k}\right|\right\}$ term.

To address this, a damped Newton search, of the form

$$
\widehat{\eta}_{i+1}=\widehat{\eta}_{i}-\alpha_{i} H_{\Gamma}^{-1}\left(\widehat{\eta}_{i}\right) g_{\Gamma}\left(\widehat{\eta}_{i}\right)
$$

is proposed where $g_{\Gamma}(\eta)$ and $H_{\Gamma}(\eta)$ are (respectively) the gradient and Hessian of $\mathcal{Q}_{\Gamma}(\eta, \widehat{R}(\eta))$ and $\alpha_{i}$ is a damping coefficient used to ensure global convergence [5]. The rationale is that since $\mathcal{Q}_{\Gamma}(\eta, \widehat{R}(\eta))$ is real valued, and hence its gradient and Hessian are real valued, then the search (43) automatically respects the realness constraint on $\widehat{\eta}$.

Of course, the reliability and speed of employing the iterative search (43) is an obvious concern, and to address this recall that a function $f: \mathbf{R}^{n} \rightarrow \mathbf{R}$ is termed 'quasiconvex' if for any $\alpha \in$ $\mathbf{R}$ the sublevel set $\{x: f(x) \leq \alpha\}$ is convex [8]. Intuitively, these functions are thus 'cone shaped', and are often termed 'unimodal' in recognition of their lack of multiple local minima [1]. Furthermore, a sufficient condition for $f$ to be quasiconvex is that

$$
f(y) \leq f(x) \quad \Longrightarrow \quad \nabla f(x)^{T}(y-x) \leq 0
$$

which implies that gradient based search, and in particular damped-Newton iterations such as (43) converge quickly and reliably [8], [5].

Lemma 4: The function $\mathcal{Q}_{\Gamma}(\eta, \widehat{R}(\eta))$ defined in (39) is quasiconvex as a function of $\eta$.

Proof: See Appendix IV.

Turning to $\mathcal{Q}_{\Delta}\left(\beta, Q, \widehat{\theta}_{k}\right)$, an identical argument and approach applies to deriving a real valued minimizer $\widehat{\beta}$ via the Newton search update of

$$
\widehat{\beta}_{i+1}=\widehat{\beta}_{i}-\delta_{i} H_{\Delta}^{-1}\left(\widehat{\beta}_{i}\right) g_{\Delta}\left(\widehat{\beta}_{i}\right)
$$

where $g_{\Delta}(\beta)$ and $H_{\Delta}(\beta)$ are (respectively) the gradient and Hessian of $\mathcal{Q}_{\Delta}\left(\beta, Q, \widehat{\theta}_{k}\right)$ and $\delta_{i}$ is again a damping coefficient, chosen to ensure global convergence. Unfortunately, because of the $\sum_{k}^{N} \operatorname{Re}\left\{\log \left|A_{k}\right|\right\}$ term, $\mathcal{Q}_{\Delta}\left(\beta, Q, \widehat{\theta}_{i}\right)$ is not, in general, quasiconvex in $\beta$, but it is nevertheless the experience of the authors (to be illustrated in a section to follow) that (45) provides a quick and reliable means for minimising $\mathcal{Q}_{\Delta}\left(\beta, Q, \widehat{\theta}_{i}\right)$.

In order to employ the damped-Newton search of (43),(45) we require the computation of the gradient and Hessian, which is detailed in the following Lemmas.

Lemma 5: The gradients for $\mathcal{Q}_{\Gamma}(\eta, \widehat{R}(\eta))$ and $\mathcal{Q}_{\Delta}(\beta, \widehat{Q}(\beta))$ are given by

$$
\begin{aligned}
& g_{\Gamma}(\eta) \triangleq \frac{\partial \mathcal{Q}_{\Gamma}(\eta, \widehat{R}(\eta))}{\partial \eta} \\
& =2 \operatorname{Re}\left\{\operatorname{vec}\left\{\widehat{R}(\eta)^{-1}[\Gamma \Sigma-\Psi]\right\}\right\}, \\
& g_{\Delta}(\beta) \triangleq \frac{\partial \mathcal{Q}_{\Delta}(\beta, \widehat{Q}(\beta))}{\partial \beta} \\
& =2 \operatorname{Re}\left\{\operatorname{vec}\left\{\widehat{Q}(\beta)^{-1}[\Delta \Sigma-\Omega]\right\}\right. \\
& \left.+\sum_{k=1}^{M}\left[\begin{array}{c}
\left.\operatorname{vec}\left\{A_{k}^{-T}\right\}\right] \\
\varnothing_{n m}
\end{array}\right]\right\}
\end{aligned}
$$

respectively.

Proof: See Appendix II.

Lemma 6: The Hessian matrices for $\mathcal{Q}_{\Gamma}(\eta, \widehat{R}(\eta))$ and $\mathcal{Q}_{\Delta}(\beta, \widehat{Q}(\beta))$ are given by

$$
\begin{aligned}
H_{\Gamma}(\eta) \triangleq & \frac{\partial^{2}}{\partial \eta \partial \eta^{T}} \mathcal{Q}_{\Gamma}(\eta, \widehat{R}(\eta)) \\
= & 2 \operatorname{Re}\left\{\Sigma \otimes \widehat{R}(\eta)^{-1}\right\} \\
& -M J_{\Gamma}^{T}(\eta)\left[\widehat{R}(\eta)^{-1} \otimes \widehat{R}(\eta)^{-T}\right] J_{\Gamma}^{C}(\eta), \\
H_{\Delta}(\beta) \triangleq & \frac{\partial^{2}}{\partial \beta \partial \beta^{T}} \mathcal{Q}_{\Delta}(\beta, \widehat{Q}(\beta)) \\
= & 2 \operatorname{Re}\left\{\Sigma \otimes \widehat{Q}(\beta)^{-1}\right\} \\
& -M J_{\Delta}^{T}(\beta)\left[\widehat{Q}(\beta)^{-1} \otimes \widehat{Q}(\beta)^{-T}\right] J_{\Delta}^{C}(\beta) \\
& +\left[\begin{array}{cc}
2 \operatorname{Re}\left(\sum_{k=1}^{M} A_{k}^{-1} \otimes A_{k}^{-T}\right) K & \varnothing_{n^{2} \times n m} \\
\varnothing_{n m \times n^{2}} & \varnothing_{n m \times n m}
\end{array}\right]
\end{aligned}
$$


where

$$
\begin{aligned}
J_{\Gamma}(\eta) & \triangleq \frac{\partial \operatorname{vec}\{\widehat{R}(\eta)\}}{\partial \eta^{T}} \\
& =\frac{1}{M}\left(\left[\Gamma \Sigma^{T}-\Psi^{C}\right] \otimes I_{p}+\left(I_{p} \otimes[\Gamma \Sigma-\Psi]\right) K\right), \\
J_{\Delta}(\beta) & \triangleq \frac{\partial \operatorname{vec}\{\widehat{Q}(\beta)\}}{\partial \beta^{T}} \\
& =\frac{1}{M}\left(\left[\Delta \Sigma^{T}-\Omega^{C}\right] \otimes I_{n}+\left(I_{n} \otimes[\Delta \Sigma-\Omega]\right) K\right)
\end{aligned}
$$

and $K$ is a commutation matrix [13, p.46], which satisfies

$$
K \operatorname{vec}\{\cdot\}=\operatorname{vec}\left\{\cdot{ }^{T}\right\}, \quad K^{T} \operatorname{vec}\left\{\cdot{ }^{T}\right\}=\operatorname{vec}\{\cdot\} \cdot
$$

Proof: See Appendix III.

\section{COMPUTATIONAL CONSIDERATIONS}

\section{A. Computing the Gradient and Hessian}

In the previous section we provided expressions for the gradients and Hessian matrices of $\mathcal{Q}_{\Gamma}(\eta, \widehat{R}(\eta))$ and $\mathcal{Q}_{\Delta}(\beta, \widehat{Q}(\beta))$.

The computational load for the M-step is dominated by an $O\left(N m^{3} n^{3}\right)$ matrix factorization and solve step for the damped-Newton method in order to obtain the search direction for estimating $A$ and $B$. As the order of the system increases to large values, this will quickly become inhibitory and iterative methods such as conjugate-gradients [19] may need to be employed.

The computational load for the E-step is dominated by computing $A_{k}=\left(\gamma_{k} I_{n}-A\right)^{-1}$ multiple times, which is an $O\left(N n^{3}\right)$ operation for $N$ measurements. However, this burden may be significantly decreased by performing an eigenvalue decomposition of $A$

$$
A V=V J
$$

where the columns of $V$ hold the eigenvectors of $A$ and $J$ is a diagonal matrix whose diagonal entries are the corresponding eigenvalues of $A$. The utility of this decomposition is that

$$
A_{k}=V\left(\gamma_{k} I_{n}-J\right) V^{-1}
$$

so that computing each $A_{k}$ is a matter of updating the diagonal matrix $\left(\gamma_{k} I_{n}-J\right)$, but possibly more importantly

$$
A_{k}^{-1}=V\left(\gamma_{k} I_{n}-J\right)^{-1} V^{-1} \text {. }
$$

Therefore, once (51) is solved once, each computation of $A_{k}^{-1}$ requires only diagonal (as opposed to full) matrix inversion. Furthermore, using the same ideas, after defining $J_{k}$ as

$$
J_{k} \triangleq\left(\gamma_{k} I_{n}-J\right)
$$

then when computing the gradient (47), the following formulation is efficient:

$$
\sum_{k=1}^{N} \operatorname{vec}\left\{A_{k}^{-T}\right\}=\operatorname{vec}\left\{V^{-T}\left[\sum_{k=1}^{N} J_{k}^{-1}\right] V^{T}\right\}
$$

as is the formulation

$$
\begin{aligned}
& \sum_{k=1}^{N}\left[A_{k}^{-1} \otimes A_{k}^{-T}\right] K \\
& \quad=\left(V \otimes V^{-T}\right)\left[\sum_{k=1}^{N}\left(J_{k}^{-1} \otimes J_{k}^{-1}\right)\right]\left(V^{-1} \otimes V^{T}\right) K
\end{aligned}
$$

for computing the Hessian (49).

\section{B. Continuous Time Modelling}

The developments to this point have been independent of the choice of the frequency domain variable $\gamma_{k}$ introduced in (1). However, the terms in (28)-(30) involve components scaled by $\gamma_{k}$ and $\left|\gamma_{k}\right|^{2}$. When fitting a continuous time model, whereby $\gamma_{k}=j \omega_{k}$, this implies scalings proportional to $\omega_{k}$ and $\omega_{k}^{2}$, which may lead to numerical problems in cases where $\omega_{k}$ varies over a large dynamic range.

This type of problem encountered with frequency domain estimation of continuous time models is widely acknowledged in the literature [20], [17]. It is routinely addressed by first scaling the measurement frequencies $\omega_{k}$ to reduce their dynamic range, fitting a model, and then scaling the model to remove the effect of 'pre-warping' the $\omega_{k}$ points. This paper proposes the same well accepted approach.

Perhaps the simplest example of this technique involves the scaling

$$
\omega_{k} \mapsto \frac{\omega_{k}}{\omega_{\text {med }}}
$$

where $\omega_{\text {med }}$ is the median value of all the points $\omega_{1}, \ldots, \omega_{N}$. The system matrices $A, B$ estimated with respect to this warped frequency axis are then themselves scaled to deliver the final estimates

$$
A \mapsto \frac{1}{\omega_{\text {med }}} A, \quad B \mapsto \frac{1}{\omega_{\text {med }}} B .
$$

While this is an effective strategy, in the authors experience, an alternative approach developed in [17] provides slightly better performance. This involves linking the desired continuous-time estimate $G(s, \theta)$ to a discrete-time one $\mathcal{G}(q, \theta)$ via the bilinear transform $\mathcal{B}(q) \triangleq \lambda(q-1) /(q+1)$, so that $\mathcal{G}(q, \theta)=G(\mathcal{B}(q), \theta)$ for the user defined constant $\lambda$.

A continuous-time estimate $G(s, \theta)$ is then obtained by first finding a discrete time estimate $\mathcal{G}(q, \theta)$ at frequency points $\omega_{k}$ scaled via

$$
\omega_{k} \mapsto \bar{\omega}_{k} \triangleq \frac{2}{T} \tan ^{-1} \frac{\omega_{k}}{\lambda}
$$

where $T$ is the underlying sampling period. Since

$$
\mathcal{B}\left(e^{j \bar{\omega}_{k} T}\right)=j \lambda \tan \frac{\bar{\omega}_{k} T}{2}=j \omega_{k}
$$

then the discrete time model estimate $\mathcal{G}\left(e^{j \bar{\omega}_{k} T}, \theta\right)$ will have identical frequency response as an associated continuous-time estimate $G\left(j \omega_{k}, \theta\right)$ if the latter is formed as

$$
G(s, \theta)=\mathcal{G}\left(\mathcal{B}^{-1}(q), \theta\right) .
$$


This inverse mapping is very simply achieved by substituting $q=\mathcal{B}^{-1}(q)=(\lambda+s) /(\lambda-s)$ to derive a mapping from discrete-time estimates $\bar{A}, \bar{B}, \bar{C}, \bar{D}$ to continuous-time model estimates $A, B, C, D$ as [17]

$$
\begin{aligned}
& A \triangleq \lambda(\bar{A}+I)^{-1}(\bar{A}-I), \quad B \triangleq \sqrt{2 \lambda}(\bar{A}+I)^{-1} \bar{B} \\
& C \triangleq \sqrt{2 \lambda} \bar{C}(\bar{A}+I)^{-1}, \quad D \triangleq \bar{D}-\bar{C}(\bar{A}+I)^{-1} \bar{B} .
\end{aligned}
$$

\section{ALGORITHM}

The preceding arguments and derivations are summarized here via a formal specification of the EM-based algorithm proposed in this paper.

Initialize $i=0$ and $\hat{\theta}_{0}$ and then perform the following steps.

1) E-STEP

a) Compute $\mathrm{E}_{\widehat{\theta}_{i}}\left\{X_{k} \mid Y_{k}\right\}$ and $\mathrm{E}_{\widehat{\theta}_{i}}\left\{X_{k} X_{k}^{\star} \mid Y_{k}\right\}$ for $k=$ $1, \ldots, N$ via Lemma 2

b) Compute $\Phi, \Psi, \Sigma, \Lambda$ and $\Omega$ via Lemma 1 ;

2) M-STEP

a) Determine a value $\eta=\widehat{\eta}_{i+1}$ that reduces $\mathcal{Q}_{\Gamma}\left(\eta, \widehat{R}(\eta), \widehat{\theta}_{i}\right)$ defined in (39) using the gradient and Hessian information from Lemmas 5 and 6 to implement the damped-Newton search (43);

b) Determine a value $\beta=\widehat{\beta}_{i+1}$ that reduces $\mathcal{Q}_{\Delta}\left(\beta, \widehat{Q}(\beta), \widehat{\theta}_{i}\right)$ defined in (40) using the gradient and Hessian information from Lemmas 5 and 6 to implement the damped-Newton search (45);

c) Compute $\widehat{R}\left(\widehat{\eta}_{i+1}\right)$ and $\widehat{Q}\left(\widehat{\beta}_{i+1}\right)$ from (33) and (35), respectively;

d) Set

$$
\widehat{\theta}_{i+1}=\left\{\widehat{\beta}_{i+1}, \widehat{\eta}_{i+1}, \operatorname{vec}\left\{\widehat{Q}\left(\widehat{\beta}_{i+1}\right)\right\}, \operatorname{vec}\left\{\widehat{R}\left(\widehat{\eta}_{i+1}\right)\right\}\right\} .
$$

3) If converged, as measured by the criterion of choice, then stop. Otherwise set $i=i+1$ and repeat.

\section{CONVERGENCE ANALYSIS}

The convergence properties of the EM algorithm derive from the fact that $\mathcal{Q}\left(\theta, \widehat{\theta}_{i}\right)$ approximates the likelihood $L(\theta)$ locally around $\widehat{\theta}_{i}$. For example, via (22), any value of $\theta$ that increases $\mathcal{Q}\left(\theta, \widehat{\theta}_{i}\right)$ must also increase $L(\theta)$. Furthermore, since

$$
\begin{aligned}
\frac{\partial}{\partial \theta} \mathcal{Q}\left(\theta, \widehat{\theta}_{i}\right) & \\
= & \frac{\partial}{\partial \theta} \int \log p_{\theta}(\bar{X}, \bar{Y}) p_{\widehat{\theta}_{i}}(\bar{X} \mid \bar{Y}) \mathrm{d} \bar{X} \\
= & \int \frac{p_{\theta}^{\prime}(\bar{X}, \bar{Y})}{p_{\theta}(\bar{X}, \bar{Y})} p_{\widehat{\theta}_{i}}(\bar{X} \mid \bar{Y}) \mathrm{d} \bar{X} \\
= & \int \frac{\partial}{\partial \theta}\left[p_{\theta}(\bar{Y}) p_{\theta}(\bar{X} \mid \bar{Y})\right] \frac{p_{\widehat{\theta}_{i}}(\bar{X} \mid \bar{Y})}{p_{\theta}(\bar{X}, \bar{Y})} \mathrm{d} \bar{X} \\
= & \int p_{\theta}^{\prime}(\bar{Y}) p_{\theta}(\bar{X} \mid \bar{Y}) \frac{p_{\widehat{\theta}_{i}}(\bar{X} \mid \bar{Y})}{p_{\theta}(\bar{X}, \bar{Y})} \mathrm{d} \bar{X} \\
& +\int p_{\theta}(\bar{Y}) p_{\theta}^{\prime}(\bar{X} \mid \bar{Y}) \frac{p_{\widehat{\theta}_{i}}(\bar{X} \mid \bar{Y})}{p_{\theta}(\bar{X}, \bar{Y})} \mathrm{d} \bar{X}
\end{aligned}
$$

$$
\begin{aligned}
= & \int \frac{p_{\theta}^{\prime}(\bar{Y})}{p_{\theta}(\bar{Y})} p_{\widehat{\theta}_{i}}(\bar{X} \mid \bar{Y}) \mathrm{d} \bar{X} \\
& +\int \frac{p_{\theta}^{\prime}(\bar{X} \mid \bar{Y})}{p_{\theta}(\bar{X} \mid \bar{Y})} p_{\widehat{\theta}_{i}}(\bar{X} \mid \bar{Y}) \mathrm{d} \bar{X} \\
= & \frac{\partial}{\partial \theta} \log p_{\theta}(\bar{Y})+\frac{\partial}{\partial \theta} \int p_{\theta}(\bar{X} \mid \bar{Y}) \frac{p_{\widehat{\theta}_{i}}(\bar{X} \mid \bar{Y})}{p_{\theta}(\bar{X} \mid \bar{Y})} \mathrm{d} \bar{X}
\end{aligned}
$$

then

$$
\left.\frac{\partial}{\partial \theta} \mathcal{Q}\left(\theta, \widehat{\theta}_{i}\right)\right|_{\theta=\widehat{\theta}_{i}}=\left.\frac{\partial}{\partial \theta} L(\theta)\right|_{\theta=\widehat{\theta}_{i}}
$$

and hence if the EM algorithm terminates at a point $\theta_{i}$ because it is a stationary point of $\mathcal{Q}\left(\theta, \theta_{i}\right)$, then it is also a stationary point of the log likelihood $L(\theta)$, or more formally:

Lemma 7: Let $\widehat{\theta}_{i+1}$ be generated from $\widehat{\theta}_{i}$ by an iteration of EM Algorithm (23), (24). Then

$$
L\left(\widehat{\theta}_{i+1}\right) \geq L\left(\widehat{\theta}_{i}\right) \quad \forall i=0,1,2, \ldots
$$

with equality if and only if both

$$
\mathcal{Q}\left(\widehat{\theta}_{i+1}, \widehat{\theta}_{i}\right)=\mathcal{Q}\left(\widehat{\theta}_{i}, \widehat{\theta}_{i}\right)
$$

and

$$
p_{\widehat{\theta}_{i+1}}(\bar{X} \mid \bar{Y})=p_{\widehat{\theta}_{i}}(\bar{X} \mid \bar{Y})
$$

for almost all (with respect to Lebesgue measure) $X$.

Proof: The proof is identical to that of Theorem 5.1 in [6].

Therefore, since by the assumptions on $\Theta$ the log likelihood $L(\theta)$ is bounded, by establishing in Lemma 7 that the likelihood values $\left\{L\left(\theta_{i}\right)\right\}$ are a monotonic sequence, the lemma also establishes that they are a convergent sequence. Furthermore, limit points of the sequence $\widehat{\theta}_{i}$ are stationary points of the log likelihood as established via the following Lemma.

Lemma 8: Let $\left\{\widehat{\theta}_{i}\right\} \subset \Theta$ be a sequence of estimates generated by the EM Algorithms (23), (24). Then a limit point $\bar{\theta}$ of $\left\{\widehat{\theta}_{i}\right\}$, is a stationary point of $L(\theta)$ and the sequence $\left\{L\left(\widehat{\theta}_{i}\right)\right\}$ converges monotonically to $L(\bar{\theta})$.

Proof: See Theorem 5.2 of [6].

A disadvantage of EM-based algorithms is that, in general, it is difficult to theoretically establish that a limit point $\bar{\theta}$ exists without imposing further (strong) conditions, such as the "classical" one imposed below.

Lemma 9: Let $\left\{\widehat{\theta}_{i}\right\} \subset \Theta$ be a sequence of estimates generated by the EM Algorithms (23), (24) and assume that there exists a $\tau>0$ such that

$$
\mathcal{Q}\left(\widehat{\theta}_{i+1}, \widehat{\theta}_{i}\right)-\mathcal{Q}\left(\widehat{\theta}_{i}, \widehat{\theta}_{i}\right) \geq \tau\left\|\widehat{\theta}_{i+1}-\widehat{\theta}_{i}\right\|^{2} .
$$

Then

$$
\lim _{i \rightarrow \infty}\left\|\widehat{\theta}_{i}-\bar{\theta}\right\|^{2}=0
$$

for some $\bar{\theta} \in \Theta$.

Proof: The proof is taken from [4], but reproduced here in order to have a self contained presentation since it is so short. 
Since $L\left(\widehat{\theta}_{i}\right)$ is a Cauchy sequence, then for any $\epsilon>0$ there exists an $N$ such that for $i \geq N$ and all $\ell \geq 1$

$$
L\left(\widehat{\theta}_{i+\ell}\right)-L\left(\widehat{\theta}_{i}\right)=\sum_{j=1}^{\ell} L\left(\widehat{\theta}_{i+j}\right)-L\left(\widehat{\theta}_{i+j-1}\right) \leq \epsilon
$$

and hence by (22) and the assumption (66)

$$
\begin{aligned}
& \tau \sum_{j=1}^{\ell}\left\|\widehat{\theta}_{i+j}-\widehat{\theta}_{i+j-1}\right\|^{2} \\
& \quad \leq \sum_{j=1}^{\ell} \mathcal{Q}\left(\widehat{\theta}_{i+j}, \widehat{\theta}_{i+j-1}\right)-\mathcal{Q}\left(\widehat{\theta}_{i+j-1}, \theta_{i+j-2}\right) \\
& \quad \leq \sum_{j=1}^{\ell} L\left(\widehat{\theta}_{i+j}\right)-L\left(\widehat{\theta}_{i+j-1}\right) \leq \epsilon
\end{aligned}
$$

which establishes (67).

\section{EXPERIMENTAL STUDY}

In this section we demonstrate the potential utility of the EM algorithm by profiling it against state-of-the-art and widely accepted gradient-based methods. To achieve this we consider a number of Monte-Carlo simulations with systems ranging from sixth-order, single-input single-output to 18th-order, three-input three-output. More precisely, three algorithms were considered, namely:

- The EM algorithm from Section IV-this is denoted EM throughout;

- A standard implementation of the Levenberg-Marquardt algorithm for non-linear least squares, where the objective is to minimize the negative log-likelihood. This is denoted as LM throughout.

- Matlab's System Identification Toolbox [12] implementation of the Levenberg-Marquardt algorithm, where the objective is also to minimize the negative log-likelihood. This is denoted as SIT throughout.

Note that the EM algorithm discussed in this paper is designed to produce ML estimates for the case of both state and measurement noise. However, the gradient-based methods mentioned above are designed to produce ML estimates but only for the case of measurement noise. Therefore, to obtain parity across the different algorithms, all simulations were conducted without state noise so that the assumed model structures and algorithms are, at least in theory, able to explain the measured data.

The first example we consider is a single-input single-output (SISO) system, whose transfer function is given by

$$
\begin{aligned}
G(s)= & \frac{2.021 \times 10^{-3}}{(s+0.1)(s+0.2)} \\
& \times \frac{1}{(s+0.01+0.1 j)(s+0.01-0.1 j)} \\
& \times \frac{1}{(s+0.02+j)(s+0.02-j)} .
\end{aligned}
$$

This system was discretized using a zero-order-hold function with one-second sample time resulting in system $\bar{G}(z)$. Output
TABLE I

COMBINATIONS OF SYSTEM ORDER AND INPUT-OUTPUT DIMENSIONS USED FOR EXPERIMENTS ON FIXED SYSTEM $\left(s_{1}\right)$ OR SUIT OF RANDOMLY CHOSEN SYSTEMS $\left(s_{2}-s_{5}\right)$

\begin{tabular}{|c|c|c|c|c|}
\hline Label & System Order & \# Inputs & \# Outputs & Type \\
\hline$s_{1}$ & $n=6$ & $m=1$ & $p=1$ & As per (70) \\
\hline$s_{2}$ & $n=2$ & $m=1$ & $p=1$ & Random \\
\hline$s_{3}$ & $n=3$ & $m=1$ & $p=1$ & Random \\
\hline$s_{4}$ & $n=8$ & $m=2$ & $p=2$ & Random \\
\hline$s_{5}$ & $n=18$ & $m=3$ & $p=3$ & Random \\
\hline
\end{tabular}

TABLE II

NUMBER OF FAILURES FOR DIFFERENT ALGORITHMS (ROWS) AND FOR DifFerent Simulations (COLUMNS) As DEFined IN TABLE I

\begin{tabular}{|c|c|c|c|c|c|}
\hline & $s_{1}$ & $s_{2}$ & $s_{3}$ & $s_{4}$ & $s_{5}$ \\
\hline EM & 0 & 2 & 4 & 8 & 13 \\
\hline LM & 0 & 5 & 7 & 12 & 28 \\
\hline SIT & 27 & 23 & 42 & 57 & 65 \\
\hline
\end{tabular}

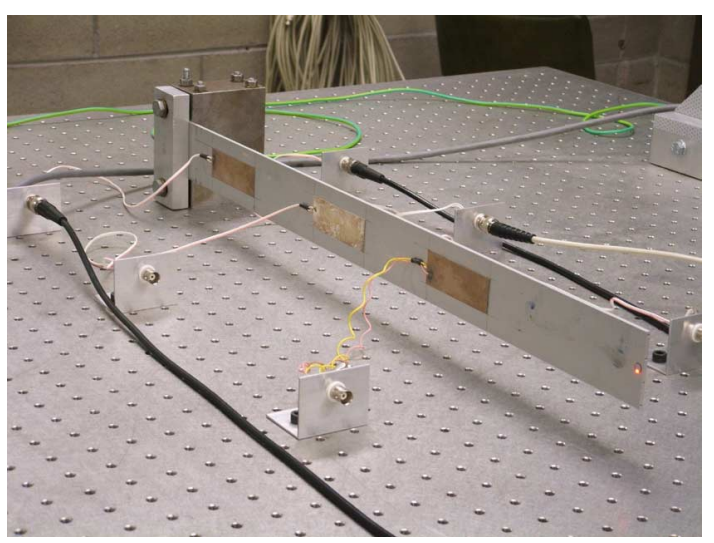

Fig. 1. Cantilevered beam experimental apparatus.

measurements $\left\{Y_{1}, \ldots, Y_{N}\right\}$, where $N=500$, were obtained via

$$
Y_{k}=\bar{G}\left(e^{j \omega_{k}}\right)+V_{k}, \quad V_{k} \sim \mathcal{N}_{c}(0,0.01)
$$

with $\omega_{k}$ being logarithmically spaced in the interval $\left[10^{-3}, \pi\right]$

We performed 100 simulations, where the noise and initial guess at parameter values were regenerated at the beginning of each run. In particular, the initial guess was randomly generated, but then ensured to correspond to a stable system.

To measure the success of each algorithm, the modelling error of the final estimate $\widehat{\theta}$, denoted $e(\widehat{\theta})$ and defined via

$$
\begin{aligned}
e(\widehat{\theta}) & \triangleq \operatorname{det}\left(\frac{1}{N} \sum_{k=1}^{N} E_{k}(\widehat{\theta}) E_{k}(\widehat{\theta})^{\star}\right), \\
E_{k}(\widehat{\theta}) & \triangleq Y_{k}-\widehat{G}\left(e^{j \omega_{k}}\right) \\
\widehat{G}\left(e^{j \omega_{k}}\right) & \triangleq \widehat{C}\left(e^{j \omega_{k}} I-\widehat{A}\right)^{-1} \widehat{B}+\widehat{D}
\end{aligned}
$$

was computed and compared with the determinant of the sample covariance

$$
v \triangleq \operatorname{det}\left(\frac{1}{N} \sum_{k=1}^{N} V_{k} V_{k}^{\star}\right)
$$




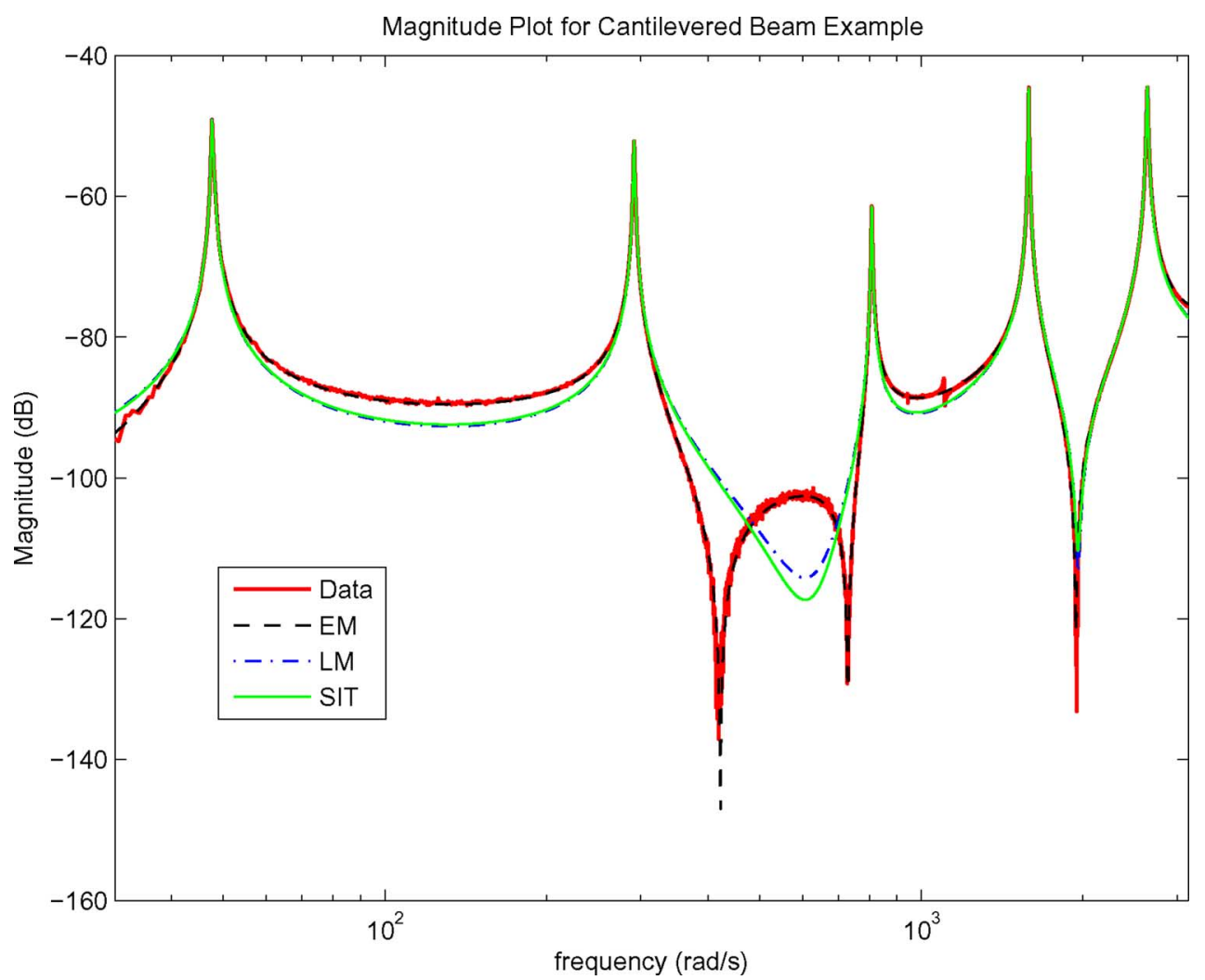

Fig. 2. Measured frequency response together with that of estimated models.

From (71) it follows that if $\widehat{\theta}$ is a good estimate then $e(\widehat{\theta}) \approx v$ is expected. With this in mind, a failure was flagged if

$$
e(\widehat{\theta})>1.3 v \text {. }
$$

Using this criteria, the number of failures for the first simulation are recorded in Table II under the heading $s_{1}$. Note that both the EM and LM approaches proved robustly successful, despite all starting from initializations which were "failures" according to the criterion (74).

In consideration of these initial encouraging results, further Monte-Carlo simulations (labelled as $s_{2}-s_{5}$ ) of randomly chosen systems of increased system dimension were conducted as described in Table I.

Each of these cases are studied in Monte-Carlo fashion by generating 100 randomly chosen systems. Different noise realizations and randomly chosen initial guesses for the parameters were also selected for each of these 100 cases. The number of estimation failures [as defined by (74)] are recorded in Table II under the columns labelled with headings $s_{2}-s_{5}$. For the lower order systems we see that EM performs slightly better than LM and both perform substantially better than SIT. As the system order is increased, the gap (in terms of number of failures) begins to grow between EM and LM indicating a clear performance advantage offered by the EM approach developed here.

While the above simulations indicate the potential utility of the EM algorithm, it is of course interesting to assess performance on a real physical system. For this purpose, a $2 \times 2$ MIMO case arising from an aluminium cantilevered beam with piezoelectric actuators and sensors as shown in Fig. 1 is considered.
Frequency domain measurements of the response of this apparatus at $N=6337$ non-uniformly spaced frequency points between $5 \mathrm{~Hz}$ and $500 \mathrm{~Hz}$ were obtained by DFT analysis of experiments involving a periodic chirp signal excitation. For this experiment, the most natural model structure is continuous rather than discrete.

Fig. 2 shows the measured frequency response for one of the transfer functions from this two-input, two-output system (the others are omitted for brevity). Fig. 2 also shows the model fit for each algorithm and Fig. 3 shows its associated error plot. In each case the system order was selected as $n=12$, with $m=2$ and $p=2$. Furthermore, each algorithm was initialized with the same parameter values as determined using a frequency-domain subspace algorithm [17].

Note that the EM algorithm clearly outperforms the gradientbased algorithms in this case in that according to Fig. 3, the modelling error is between 10 and $30 \mathrm{~dB}$ smaller when employing the EM approach. In particular, note from Fig. 2 how the EM method is able to accurately model two lightly damped zeros between 40 and $80 \mathrm{rad} / \mathrm{s}$ that are completely missed by both gradient search based implementations considered here. Importantly, not capturing these modelling aspects can have significant implications for the success of subsequent feedback control design [24].

As a final test, again using data from a real physical system, the "vibrating mechanical system" profiled in [22] is considered using the measurement data and associated report available at elecftp.vub.ac.be in the subdirectory/Pub/IdentificationData/Report1998nr3. This data consists of 102400 time domain measurements. These were processed by a standard 


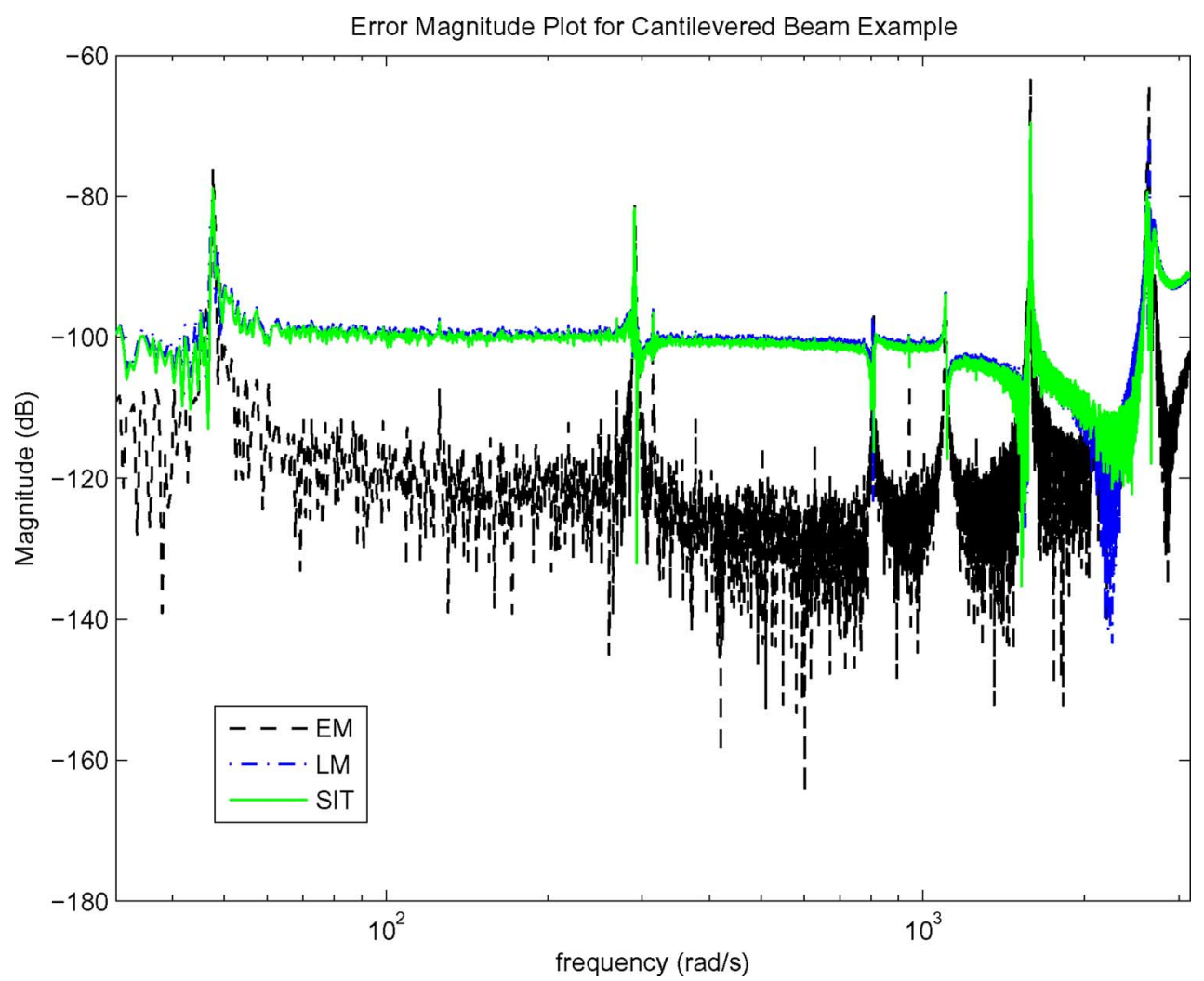

Fig. 3. Modelling error associated with the estimates shown in Fig. 2.

Blackman-Tukey method with full width Hamming window to provide $N=641$ frequency response measurements in the range from 95 to $6200 \mathrm{~Hz}$ [23].

Fig. 4 shows the associated nonparametric frequency response measurements (solid line) together with (dashed line) a 23rd order model obtained via the EM algorithm described in Section VIII. The EM method was initialized with a subspace-based estimate [17] shown as a dash-dot line. Note that this subspace estimate was deliberately chosen to be quite "poor" in order to show that the EM algorithm converges from such initial estimates. With some tuning, the subspace algorithm should perform much better than this.

Again, despite the high model order, wide bandwidth over which a fit is required, and wide dynamic range of measurements, a very close fit between measurements and state space model response is achieved. Indeed, the fitting error, shown as a dotted line in Fig. 4 is as small, and in some frequency regions smaller that that illustrated in [22].

\section{EXTENSIONS}

As detailed in Section II, the developments in this paper have concentrated on the situation where measurements of the frequency response function are available. This involves the $i, \ell$ th element of $Y\left(\omega_{k}\right) \in \mathbf{C}^{p \times m}$ being the magnitude and phase response at frequency $\omega_{k}$ (in phasor form) of the $i$ th output to the $\ell$ th input and, in turn, the $i$, $\ell$ th element of $U\left(\omega_{k}\right) \in \mathbf{C}^{m \times m}$ being the measured magnitude and phase at frequency $\omega_{k}$ (again in phasor form) of the $i$ th input on the $\ell$ th experiment.

This format has been assumed because it so commonly occurs in practice, principally by the design of commercial equipment specifically targetted at obtaining frequency response function measurements.

An important alternative scenario is one whereby available measurements are the discrete Fourier transforms (DFT's) of the observed time domain output $y_{t} \in \mathbf{R}^{p}$ and input $u_{t} \in \mathbf{R}^{m}$. In the SISO case of $p=m=1$, then since this paper does not constrain $U\left(\omega_{k}\right)$ to be the identity, and since only one experiment is involved, this situation also fits within the assumed measurement frameork of this paper. Furthermore, with the definition of the $N$ point DFT $Y(\omega)$ of the time domain signal $y_{t}$ as

$$
Y(\omega)=\frac{1}{\sqrt{N}} \sum_{t=1}^{N} y_{t} e^{-j \omega t}
$$

then with the definition $I_{x}(\omega)=N^{-1 / 2}\left(x_{N+1} e^{-j \omega N}-x_{1}\right)$ the first row of the model structure (2) becomes

$$
\begin{aligned}
e^{j \omega_{k}} X_{k} & =A X_{k}+B U_{k}+I_{x}\left(\omega_{k}\right)+W_{k} \\
& =A X_{k}+\widetilde{B} \widetilde{U}_{k}+W_{k}
\end{aligned}
$$

where $\widetilde{B}=\left[B, I_{x}\left(\omega_{k}\right)\right]$ and $\widetilde{U}_{k}=\left[U_{k}^{T}, 1\right]^{T}$. With the further (commonly occuring) specialization that the frequency points satisfy $\omega_{k}=2 \pi k / N$, then $I_{x}$ is real valued and frequency independent. This implies that by using the augmented $\widetilde{B}$ and $\widetilde{U}_{k}$ in (77), any distorting effects of non-periodic inputs can be avoided by estimating the term $I_{x}$ accounting for them. This will involve minor but straightforward modifications to the maximization step profiled in Section VI. In the more general MIMO case, this same extension allowing for estimation of edge effects can still be applied. However, more extensive (but still straightforward) 


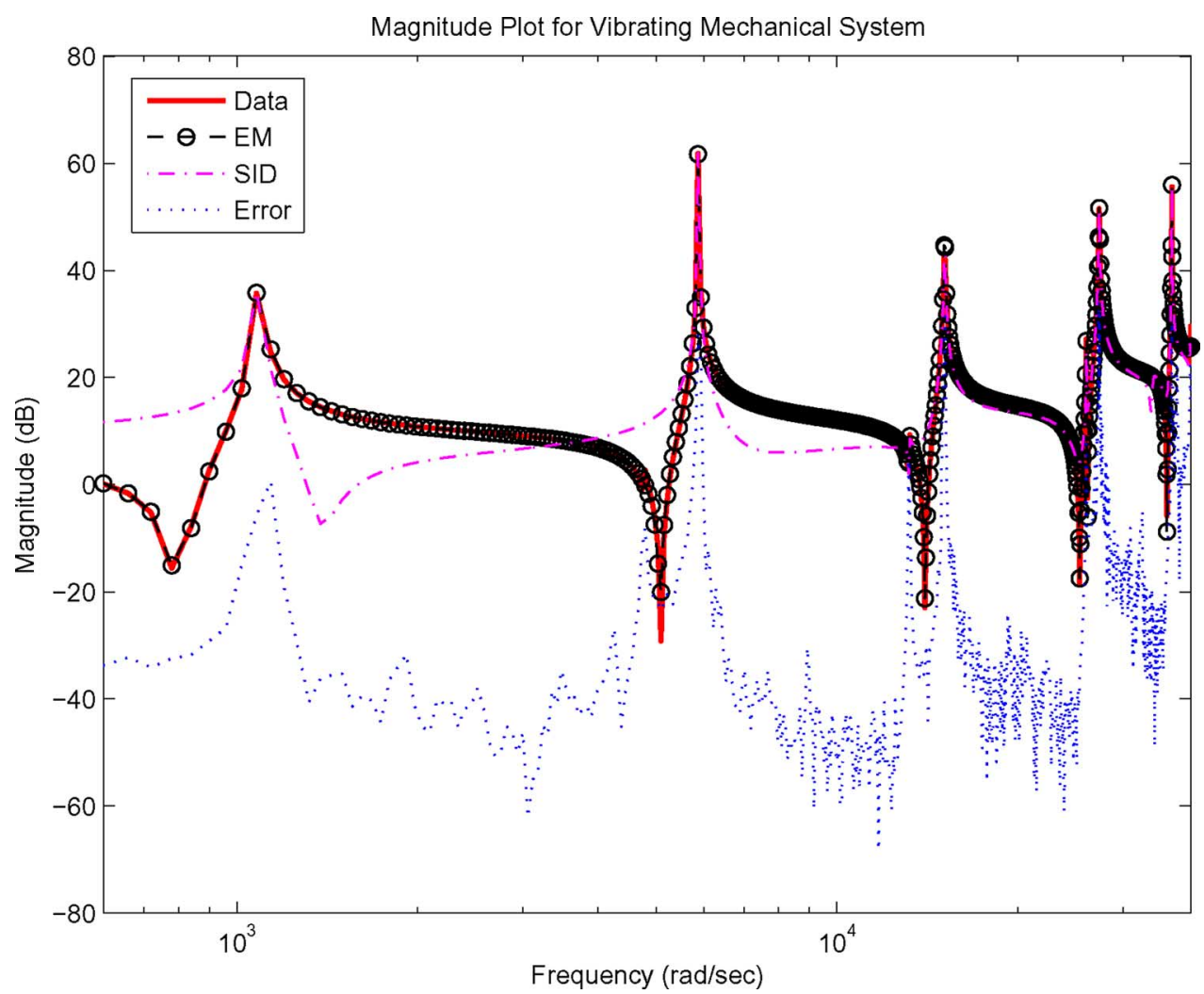

Fig. 4. Measured frequency response together with that of estimated models and modelling error for "vibrating mechanical system."

modifications will be required. For example, the model(3) will need to be altered by removing the Kronecker product terms, and the flow on effects of this in the E-step and M-step will need to be accounted for.

\section{CONCLUSION}

This paper has profiled a new method for estimation from frequency domain data that is based on the EM algorithm. As illustrated empirically, the new technique developed here has the advantages of robustness to capture in local minima and the ability to more accurately estimate higher dimensional systems (possibly over wide bandwidths) than conventional gradient based search approaches. Planned future work by the authors will be directed at analyzing and understanding the convergence properties of the method and the factors that influence convergence rate.

Readers interested in evaluating the methods presented here are invited to download the MATLAB-based system identification toolbox available at sigpromu.org/idtoolbox which implements them.

\section{APPENDIX I \\ PROOF OF LEMMA 1}

Proof: By using the fact that $Y_{k}$ is independent of $Y_{\ell}$ for $k \neq \ell$ and similarly for $X_{k}$ and $X_{\ell}$ then according to the definition of conditional probability

$$
p_{\theta}\left(Y_{1}, \ldots, Y_{N}, X_{1}, \ldots, X_{N}\right)=\prod_{k=1}^{N} p_{\theta}\left(Y_{k}, X_{k}\right)
$$

$$
=\prod_{k=1}^{N} p_{\theta}\left(Y_{k} \mid X_{k}\right) p_{\theta}\left(X_{k}\right)
$$

The above densities are given by

$$
\begin{aligned}
& p_{\widehat{\theta}_{i}}\left(Y_{k} \mid X_{k}\right)=\frac{1}{\pi^{m p} \operatorname{det}\left(I_{m} \otimes R\right)} \\
& \times \exp \left\{-\left\|\left(I_{m} \otimes R\right)^{-1 / 2} \operatorname{vec}\left\{Y_{k}-\Gamma Z_{k}\right\}\right\|^{2}\right\}
\end{aligned}
$$

and

$$
\begin{aligned}
& p_{\widehat{\theta}_{i}}\left(X_{k}\right)=\frac{1}{\pi^{m n} \operatorname{det}\left(I_{m} \otimes A_{k}^{-1} Q A_{k}^{-\star}\right)} \\
& \quad \times \exp \left\{-\left\|\left(I_{m} \otimes A_{k}^{-1} Q A_{k}^{-\star}\right)^{-1 / 2} \operatorname{vec}\left\{X_{k}-A_{k}^{-1} B\right\}\right\|^{2}\right\} .
\end{aligned}
$$

Using the fact that $\left(I_{m} \otimes R\right)$ is block diagonal then $\operatorname{det}\left(I_{m} \otimes\right.$ $R)=\operatorname{det}(R)^{m}$. Furthermore, with $V_{k} \triangleq Y_{k}-\Gamma Z_{k}, \widetilde{V}_{k} \triangleq$ $\operatorname{vec}\left\{V_{k}\right\}$, and $V_{k}^{i}$ being the $i$ th column of $V_{k}$ then

$$
\begin{aligned}
\left\|\left(I_{m} \otimes R\right)^{-1 / 2} \widetilde{V}_{k}\right\|^{2} & =\widetilde{V}_{k}^{\star}\left(I_{m} \otimes R\right)^{-1} \widetilde{V}_{k} \\
& =\sum_{i=1}^{m}\left\{V_{k}^{i}\right\}^{\star} R^{-1} V_{k}^{i} \\
& =\operatorname{Tr}\left\{\sum_{i=1}^{m} R^{-1} V_{k}^{i}\left\{V_{k}^{i}\right\}^{\star}\right\} \\
& =\operatorname{Tr}\left\{R^{-1} V_{k} V_{k}^{\star}\right\} .
\end{aligned}
$$


Therefore

$$
\begin{aligned}
& -\sum_{k=1}^{N} \log p_{\theta}\left(Y_{k} \mid X_{k}\right)=m N \log |R|+ \\
& \quad \sum_{k=1}^{N} \operatorname{Tr}\left\{R^{-1}\left(Y_{k}-\Gamma Z_{k}\right)\left(Y_{k}-\Gamma Z_{k}\right)^{\star}\right\} .
\end{aligned}
$$

Applying the conditional expectation operator $\mathrm{E}_{\widehat{\theta}_{i}}\left\{\cdot \mid Y_{k}\right\}$ to both sides then provides the expression (26) for $\mathcal{Q}_{\Gamma}\left(\eta, R, \widehat{\theta}_{i}\right)$ with the expression (27) for $\mathcal{Q}_{\Delta}\left(\beta, Q, \widehat{\theta}_{i}\right)$ being obtained in an identical manner.

\section{APPENDIX II \\ PROOF OF LEMMA 5}

Proof: Substituting (33) into(26) provides

$$
\mathcal{Q}_{\Gamma}(\eta, \widehat{R}(\eta))=m N \log |\widehat{R}(\eta)|+c
$$

where $c$ is a constant. Direct application of Lemma 14 in Appendix $\mathrm{V}$ results in the expression $(46)$ for $g_{\Gamma}(\eta)$. In a similar manner, substituting (35) into $\mathcal{Q}_{\Delta}(\beta, Q)$ provides

$$
\begin{aligned}
\mathcal{Q}_{\Delta}(\beta, \widehat{Q}(\beta)) & =f_{1}(\beta)+f_{2}(\beta), \\
f_{1}(\beta) & \triangleq m N \log |\widehat{Q}(\beta)|+c, \\
f_{2}(\beta) & \triangleq-m \sum_{k=1}^{N}\left(\log \left|A_{k}\right|+\log \left|A_{k}^{\star}\right|\right)
\end{aligned}
$$

so that again using Lemma 14 in Appendix V

$$
\frac{\partial f_{1}(\beta)}{\partial \beta}=2 \operatorname{Re}\left\{\operatorname{vec}\left\{\widehat{Q}(\beta)^{-1}[\Delta \Sigma-\Omega]\right\}\right\} .
$$

Furthermore, by Lemma 13

$$
\begin{aligned}
\frac{\partial f_{2}(\beta)}{\partial \beta}=-m \sum_{k=1}^{N} & {\left[\frac{\partial \operatorname{vec}\left\{A_{k}\right\}}{\partial \beta^{T}}\right]^{T} \operatorname{vec}\left\{A_{k}^{-T}\right\} } \\
& -m \sum_{k=1}^{N}\left[\frac{\partial \operatorname{vec}\left\{A_{k}^{\star}\right\}}{\partial \beta^{T}}\right]^{T} \operatorname{vec}\left\{A_{k}^{-C}\right\}
\end{aligned}
$$

where

$$
\frac{\partial \operatorname{vec}\left\{A_{k}\right\}}{\partial \beta^{T}}=\frac{\partial \operatorname{vec}\left\{e^{j \omega_{k}} I-A\right\}}{\partial \beta^{T}}=\left[\begin{array}{ll}
-I & \varnothing_{n^{2} \times n m}
\end{array}\right]
$$

and ( $K$ is a commutation matrix defined in (134))

$$
\frac{\partial \operatorname{vec}\left\{A_{k}^{\star}\right\}}{\partial \beta^{T}}=\frac{\partial \operatorname{vec}\left\{e^{-j \omega_{k}} I-A^{T}\right\}}{\partial \beta^{T}}=\left[\begin{array}{ll}
-K & \varnothing_{n^{2} \times n m}
\end{array}\right]
$$

so that

$$
\begin{aligned}
\frac{\partial f_{2}(\beta)}{\partial \beta} & =m \sum_{k=1}^{N}\left[\begin{array}{c}
\operatorname{vec}\left\{A_{k}^{-T}\right\} \\
\varnothing_{n m}
\end{array}\right]+\left[\begin{array}{c}
K^{T} \operatorname{vec}\left\{A_{k}^{-C}\right\} \\
\varnothing_{n m}
\end{array}\right] \\
& =2 m \operatorname{Re}\left\{\sum_{k=1}^{N}\left[\begin{array}{c}
\operatorname{vec}\left\{A_{k}^{-T}\right\} \\
\varnothing_{n m}
\end{array}\right]\right\} .
\end{aligned}
$$

\section{APPENDIX III \\ PROOF OF LEMMA 6}

Proof: The expression (48) for the Hessian with respect to $\eta$ is established by direct application of Lemma 14 in Appendix V. To establish (49) recall the definitions of $f_{1}$ and $f_{2}$ made in (90), (91) respectively which allow the Hessian with respect to $\beta$ to be written as

$$
\frac{\partial^{2} \mathcal{Q}_{\Delta}(\beta, \widehat{Q}(\beta))}{\partial \beta \partial \beta^{T}}=\underbrace{\frac{\partial^{2} f_{1}(\beta)}{\partial \beta \partial \beta^{T}}}_{H_{1}(\beta)}+\underbrace{\frac{\partial^{2} f_{2}(\beta)}{\partial \beta \partial \beta^{T}}}_{H_{2}(\beta)} .
$$

Use of Lemma 14 then provides

$$
\begin{aligned}
H_{1}(\beta)=2 \operatorname{Re} & \left\{\Sigma \otimes \widehat{Q}(\beta)^{-1}\right\} \\
- & m N J_{\Delta}^{T}(\beta)\left[\widehat{Q}(\beta)^{-1} \otimes \widehat{Q}(\beta)^{-T}\right] J_{\Delta}^{C}(\beta) .
\end{aligned}
$$

Furthermore

$$
\begin{aligned}
& H_{2}(\beta) \\
& =\frac{\partial}{\partial \beta}\left[\frac{\partial f_{2}(\beta)}{\partial \beta}\right]^{T} \\
& =m \frac{\partial}{\partial \beta}\left(\sum_{k=1}^{N}\left[\operatorname{vec}\left\{A_{k}^{-T}\right\}^{T} \quad \varnothing_{1 \times n m}\right]\right. \\
& \left.+\left[\operatorname{vec}\left\{A_{k}^{-\star}\right\}^{T} \quad \varnothing_{1 \times n m}\right]\right) \\
& =m \sum_{k=1}^{N}\left[\begin{array}{cc}
\frac{\partial \operatorname{vec}\left\{A_{k}^{-1}\right\}^{T}}{\partial \beta}+\frac{\partial \operatorname{vec}\left\{A_{k}^{\star}\right\}^{T}}{\partial \beta} & \varnothing_{n^{2} \times n m} \\
\varnothing_{n m \times n^{2}} & \varnothing_{n m \times n m}
\end{array}\right] \\
& =-m \sum_{k=1}^{N}\left[\begin{array}{cc}
Z_{k} & \varnothing_{n^{2} \times n m} \\
\varnothing_{n m \times n^{2}} & \varnothing_{n m \times n m}
\end{array}\right] \\
& =m \sum_{k=1}^{N}\left[\begin{array}{cc}
\left(A_{k}^{-1} \otimes A_{k}^{-T}\right) K+\left(A_{k}^{-C} \otimes A_{k}^{-*}\right) K & \varnothing_{n^{2} \times n m} \\
\varnothing_{n m \times n^{2}} & \varnothing_{n m \times n m}
\end{array}\right]
\end{aligned}
$$

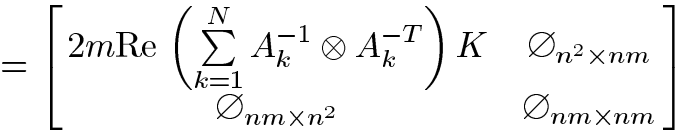

where

$Z_{k} \triangleq\left(A_{k}^{-1} \otimes A_{k}^{-T}\right) \frac{\partial \operatorname{vec}\left\{A_{k}^{T}\right\}}{\partial \beta^{T}}+\left(A_{k}^{-C} \otimes A_{k}^{-*}\right) \frac{\partial \operatorname{vec}\left\{A_{k}^{*}\right\}}{\partial \beta^{T}}$.

\section{APPENDIX IV \\ PROOF OF LEMMA 4}

Proof: Define, for a matrix $X$ of suitable size, $x \triangleq \operatorname{vec}\{X\}$ and

$$
f(x) \triangleq \mathcal{Q}_{\Gamma}(x, \widehat{R}(x))
$$


with the latter defined in (39) so that

$$
\begin{aligned}
& f(x)=m N \\
& \quad \cdot \log \operatorname{det}\left\{\frac{1}{m N}\left[\Phi-X \Psi^{\star}-\Psi X^{T}+X \Sigma X^{T}\right]\right\}+m p N .
\end{aligned}
$$

Let $y \triangleq \operatorname{vec}\{Y\}$, then $f(y) \leq f(x)$ is equivalent to (since $\log (\cdot)$ is monotonic)

$$
\begin{aligned}
\operatorname{det}\{ & \left.\frac{1}{m N}\left[\Phi-Y \Psi^{\star}-\Psi Y^{T}+Y \Sigma Y^{T}\right]\right\} \\
& \leq \operatorname{det}\left\{\frac{1}{m N}\left[\Phi-X \Psi^{\star}-\Psi X^{T}+X \Sigma X^{T}\right]\right\} .
\end{aligned}
$$

However, since the arguments for $\operatorname{det}(\cdot)$ are positive definite and Hermitian, then according to [9, Theorem 7.2.5] a necessary and sufficient condition for (103) is

$\Phi-Y \Psi^{\star}-\Psi Y^{T}+Y \Sigma Y^{T} \preceq \Phi-X \Psi^{\star}-\Psi X^{T}+X \Sigma X^{T}$

where $\preceq$ denotes the usual partial ordering on positive semidefinite matrices [9]. In turn, (104) is equivalent to

$$
Y \Sigma Y^{T}-X \Sigma X^{T} \preceq Y \Psi^{\star}+\Psi Y^{T}-X \Psi^{\star}-\Psi X^{T}
$$

Considering now the right hand side of (44), Lemma 5 provides an expression for the gradient of $f$ as

$$
\nabla f(x)=2 \operatorname{Re}\left(\operatorname{vec}\left\{\widehat{R}^{-1}(x)[X \Sigma-\Psi]\right\}\right)
$$

so that

$$
\begin{aligned}
\nabla f(x)^{T} & y-x) \\
= & 2 \operatorname{Re}\left(\operatorname{vec}\left\{\widehat{R}^{-1}(x)[X \Sigma-\Psi]\right\}\right)^{T}(y-x), \\
= & \left(\operatorname{vec}\left\{\widehat{R}^{-1}(x)[X \Sigma-\Psi]\right\}\right)^{T}(y-x) \\
& +\left(\operatorname{vec}\left\{\widehat{R}^{-T}(x)\left[X \Sigma^{T}-\Psi^{C}\right]\right\}\right)^{T}(y-x) \\
= & \operatorname{Tr}\left\{[X \Sigma-\Psi]^{\star} \widehat{R}^{-1}(x)(Y-X)\right\} \\
& +\operatorname{Tr}\left\{(Y-X)^{T} \widehat{R}^{-1}(x)[X \Sigma-\Psi]\right\} \\
= & \operatorname{Tr}\left\{\widehat{R}^{-1}(x)(Y-X)[X \Sigma-\Psi]^{\star}\right\} \\
& +\operatorname{Tr}\left\{\widehat{R}^{-1}(x)[X \Sigma-\Psi](Y-X)^{T}\right\} \\
= & \operatorname{Tr}\left\{\widehat { R } ^ { - 1 } ( x ) \left[Y \Sigma X^{T}+X \Sigma Y^{T}-2 X \Sigma X^{T}\right.\right. \\
& \left.\left.-Y \Psi^{\star}-\Psi Y^{T}+X \Psi^{\star}+\Psi X^{T}\right]\right\} .
\end{aligned}
$$

Since $\widehat{R}^{-1}(x)$ is positive definite, then $\nabla f(x)^{T}(y-x) \leq 0$ if $\quad$ where and only if

$Y \Sigma X^{T}+X \Sigma Y^{T}-2 X \Sigma X^{T}-Y \Psi^{\star}-\Psi Y^{T}+X \Psi^{\star}+\Psi X^{T} \preceq 0$.
However

$$
\begin{aligned}
0 & \preceq(Y-X) \Sigma(Y-X)^{T} \\
& =Y \Sigma Y^{T}+X \Sigma X^{T}-Y \Sigma X^{T}-X \Sigma Y^{T}
\end{aligned}
$$

which can be rewritten as

$$
Y \Sigma X^{T}+X \Sigma Y^{T}-2 X \Sigma X^{T} \preceq Y \Sigma Y^{T}-X \Sigma X^{T}
$$

so that a sufficient condition for (107) to hold is that

$$
Y \Sigma Y^{T}-X \Sigma X^{T}-Y \Psi^{\star}-\Psi Y^{T}+X \Psi^{\star}+\Psi X^{T} \preceq 0 .
$$

However, as just established via (105), this is a consequence of the assumption that $f(y) \leq f(x)$ which completes the proof by using the property (44).

APPENDIX V

AUXILIARY RESULTS

Lemma 10: Suppose

$$
\left[\begin{array}{l}
X \\
Y
\end{array}\right] \sim \mathcal{N}\left(\left[\begin{array}{l}
\mu_{X} \\
\mu_{Y}
\end{array}\right],\left[\begin{array}{cc}
P_{X} & P_{X Y} \\
P_{Y X} & P_{Y}
\end{array}\right]\right)
$$

then

$$
(X \mid Y) \sim \mathcal{N}\left(\mu_{X \mid Y}, P_{X \mid Y}\right)
$$

where

$$
\begin{aligned}
& \mu_{X \mid Y} \triangleq \mu_{X}+P_{X Y} P_{Y}^{-1}\left(Y-\mu_{Y}\right), \\
& P_{X \mid Y} \triangleq P_{X}-P_{X Y} P_{Y}^{-1} P_{Y X} .
\end{aligned}
$$

Proof: See Theorem 2.13 of [10].

Lemma 11: Let $n>0, X \in \mathbf{C}^{n \times 1}, A, B \in \mathbf{C}^{n \times n}$ be arbitrary save that $A$ is invertible. Define the operator [[- $]]$ acting on vectors and matrices such as these via

$$
[[X]] \triangleq\left[\begin{array}{c}
\operatorname{Re}\{X\} \\
\operatorname{Im}\{X\}
\end{array}\right], \quad[[A]] \triangleq\left[\begin{array}{cc}
\operatorname{Re}\{A\} & -\operatorname{Im}\{A\} \\
\operatorname{Im}\{A\} & \operatorname{Re}\{A\}
\end{array}\right]
$$

Then

$$
\begin{aligned}
& {[[A+B]]=[[A]]+[[B]], \quad[[A]][[B]]=[[A B]]} \\
& {[[A]][[X]]=[[A X]], \quad\left[\left[A^{-1}\right]\right]=[[A]]^{-1} .}
\end{aligned}
$$

Proof: See [21, Lemma 13.3].

Lemma 12: Suppose

$$
\left[\begin{array}{l}
X \\
Y
\end{array}\right] \sim \mathcal{N}_{c}\left(\left[\begin{array}{l}
\mu_{X} \\
\mu_{Y}
\end{array}\right],\left[\begin{array}{cc}
P_{X} & P_{X Y} \\
P_{Y X} & P_{Y}
\end{array}\right]\right)
$$

then

$$
(X \mid Y) \sim \mathcal{N}_{c}\left(\mu_{X \mid Y}, P_{X \mid Y}\right)
$$

$$
\begin{aligned}
& \mu_{X \mid Y} \triangleq \mu_{X}+P_{X Y} P_{Y}^{-1}\left(Y-\mu_{Y}\right) \\
& P_{X \mid Y} \triangleq P_{X}-P_{X Y} P_{Y}^{-1} P_{Y X}
\end{aligned}
$$


Proof: While this result is expected given Lemma 10, it is by no means trivially obvious given the covariance restrictions (4) necessary for a complex Gaussian distribution. Furthermore, we have been unable to find a statement, (let alone proof) of this result in the literature, and so provide it here since it is central to the development. We thank an anonymous reviewer for providing the proof below, which is much more streamlined than our first drafted version.

According to the complex normal specification (4) and the operator $[[\cdot]]$ defined in Lemma 11

$$
\left[\begin{array}{c}
[[X]]] \\
{[[Y]}
\end{array}\right] \sim \mathcal{N}\left(\left[\begin{array}{c}
{\left[\left[\mu_{X}\right]\right]} \\
{\left[\left[\mu_{Y}\right]\right.}
\end{array}\right], \Sigma\right)
$$

where

$$
\Sigma \triangleq \frac{1}{2}\left[\begin{array}{cc}
{\left[\left[P_{X}\right]\right]} & {\left[\left[P_{X Y}\right]\right.} \\
{\left[\left[P_{Y X}\right]\right]} & {\left[\left[P_{Y}\right]\right.}
\end{array}\right] .
$$

Applying Lemma 10 then yields

$$
([[X]] \mid[[Y]]) \sim \mathcal{N}(\mu, P)
$$

where

$$
\mu \triangleq\left[\left[\mu_{X}\right]\right]+\left[\left[P_{X Y}\right]\right]\left[\left[P_{Y}\right]\right]^{-1}\left([[Y]]-\left[\left[\mu_{Y}\right]\right]\right)
$$

and

$$
P \triangleq \frac{1}{2}\left[\left[P_{X}\right]\right]-\frac{1}{2}\left[\left[P_{X Y}\right]\right]\left[\left[P_{Y}\right]\right]^{-1}\left[\left[P_{Y X}\right]\right] .
$$

Applying Lemma 11 repeatedly to (122) then delivers

$$
\begin{aligned}
\mu & =\left[\left[\mu_{X}\right]\right]+\left[\left[P_{X Y}\right]\right]\left[\left[P_{Y}^{-1}\right]\right]\left([[Y]]-\left[\left[\mu_{Y}\right]\right]\right) \\
& =\left[\left[\mu_{X}\right]\right]+\left[\left[P_{X Y} P_{Y}^{-1}\right]\right]\left(\left[[Y]-\left[\left[\mu_{Y}\right]\right]\right)\right. \\
& =\left[\left[\mu_{X}+P_{X Y} P_{Y}^{-1}\left(Y-\mu_{Y}\right)\right]\right] .
\end{aligned}
$$

A similar repeated application of Lemma 11 to (123) provides

$$
P=\frac{1}{2}\left[\left[P_{X}-P_{X Y} P_{Y}^{-1} P_{Y X}\right]\right] .
$$

Therefore, with the formulations (118) and (119) for mean and covariance, the real and imaginary parts of $X \mid Y$ satisfy the distributional conditions (4) necessary for the complex valued $X \mid Y$ to obey a complex normal density.

Lemma 13: Suppose $M, N \in \mathbf{R}^{n \times n}$ and $M$ is invertible. Then

$$
\begin{gathered}
\frac{\partial}{\partial M} \log |M|=M^{-T}, \frac{\partial}{\partial M} \operatorname{Tr}\left\{M^{-1} N\right\}=-M^{-T} N^{T} M^{-T} \\
\text { and } \\
\frac{\partial}{\partial M} \operatorname{Tr}\{M N\}=N^{T}, \frac{\operatorname{dvec}\left\{M^{-1}\right\}}{\operatorname{dvec}\{M\}^{T}}=-M^{-T} \otimes M^{-1} .
\end{gathered}
$$

Proof: See [14].

Lemma 14: Suppose $X \in \mathbf{R}^{p \times q}, x \triangleq \operatorname{vec}\{X\}, A=A^{*} \in$ $\mathbf{C}^{p \times p}, B \in \mathbf{C}^{p \times q}, C=C^{*} \in \mathbf{C}^{q \times q}, \alpha>0$ and a function $f$ is defined as

$$
f(x) \triangleq \alpha \log |D(x)|
$$

$$
D(x) \triangleq \frac{1}{\alpha}\left(A-X B^{*}-B X^{T}+X C X^{T}\right) .
$$

Then

$$
\frac{\partial f(x)}{\partial x}=2 \operatorname{Re}\left\{D^{-1}(x)(X C-B)\right\}
$$

and

$$
\begin{aligned}
\frac{\partial^{2} f(x)}{\partial x \partial x^{T}}= & 2 \operatorname{Re}\left\{C \otimes D^{-1}(x)\right\}- \\
& \alpha E^{T}(x)\left[D^{-1}(x) \otimes D^{-T}(x)\right] E^{C}(x)
\end{aligned}
$$

where

$$
\begin{aligned}
E(x) & \triangleq \frac{\partial \operatorname{vec}\{D(x)\}}{\partial x^{T}} \\
& =\frac{1}{\alpha}\left(\left[X C^{T}-B^{C}\right] \otimes I_{p}+\left(I_{p} \otimes[X C-B]\right) K\right)
\end{aligned}
$$

and $K$ is a commutation matrix, which satisfies

$$
K \operatorname{vec}\{X\}=\operatorname{vec}\left\{X^{T}\right\}, \quad K^{T} \operatorname{vec}\left\{X^{T}\right\}=\operatorname{vec}\{X\} .
$$

Proof: By Lemma 13

$$
\frac{\partial f(x)}{\partial x}=\alpha\left[\frac{\partial \operatorname{vec}\{D(x)\}}{\partial x^{T}}\right]^{T} \operatorname{vec}\left\{D(x)^{-T}\right\}
$$

where using the identity vec $\{W V Z\}=\left(Z^{T} \otimes W\right) \operatorname{vec}\{V\}$

$$
\begin{aligned}
& \frac{\partial \operatorname{vec}\{D(x)\}}{\partial x^{T}} \\
= & \frac{1}{\alpha} \frac{\partial}{\partial x^{T}}\left[\operatorname{vec}\{A\}-\operatorname{vec}\left\{X B^{\star}\right\}-\operatorname{vec}\left\{B X^{T}\right\}+\operatorname{vec}\left\{X C X^{T}\right\}\right], \\
= & \frac{1}{\alpha}\left(-\left(B^{C} \otimes I\right) \frac{\partial \operatorname{vec}\{X\}}{\partial x^{T}}-(I \otimes B) \frac{\partial \operatorname{vec}\left\{X^{T}\right\}}{\partial x^{T}}\right) \\
& +\frac{1}{\alpha}\left(\left(X C^{T} \otimes I\right) \frac{\partial \operatorname{vec}\{X\}}{\partial x^{T}}+(I \otimes X C) \frac{\partial \operatorname{vec}\left\{X^{T}\right\}}{\partial x^{T}}\right) \\
= & -\frac{1}{\alpha}\left\{\left(B^{C} \otimes I\right)-(I \otimes B) K+\left(X C^{T} \otimes I\right)+(I \otimes X C) K\right\} \\
= & \frac{1}{\alpha}\left\{\left[X C^{T}-B^{C}\right] \otimes I+(I \otimes[X C-B]) K\right\}
\end{aligned}
$$

where $(\cdot)^{C}$ denotes complex conjugation and $K$ is the commutation matrix defined by

$$
K \operatorname{vec}\{X\}=\operatorname{vec}\left\{X^{T}\right\} .
$$

Substitution of (140) into (135) then delivers

$$
\begin{aligned}
\frac{\partial f(x)}{\partial x}= & {\left[\left(\left[X^{T} C-B^{\star}\right] \otimes I\right)\right.} \\
& \left.+K^{T}\left(I \otimes\left[C^{T} X^{T}-B^{T}\right]\right)\right] \operatorname{vec}\left\{D(x)^{-T}\right\} \\
= & \operatorname{vec}\left\{D(x)^{-T}\left[X C^{T}-B^{C}\right]\right\} \\
& +K^{T} \operatorname{vec}\left\{\left[C^{T} X^{T}-B^{T}\right] D(x)^{-T}\right\} \\
= & \operatorname{vec}\left\{D(x)^{-T}\left[X C^{T}-B^{C}\right]+D(x)^{-1}[X C-B]\right\}
\end{aligned}
$$




$$
=2 \operatorname{Re}\left\{\operatorname{vec}\left\{D(x)^{-1}[X C-B]\right\}\right\}
$$

where the facts that both $D(x)$ and $C$ are Hermitian was used in progressing to the last line.

Using the expression (143)

$$
\begin{aligned}
& \frac{\partial^{2} f(x)}{\partial x \partial x^{T}} \\
& =\frac{\partial}{\partial x^{T}} \operatorname{vec}\left\{D(x)^{-T}\left[X C^{T}-B^{C}\right]+D(x)^{-1}[X C-B]\right\} \\
& =\operatorname{vec}\left\{D(x)^{-T} \frac{\partial\left[X C^{T}-B^{C}\right]}{\partial x^{T}}+D(x)^{-1} \frac{\partial[X C-B]}{\partial x^{T}}\right\} \\
& +\operatorname{vec}\left\{\frac{\partial D(x)^{-T}}{\partial x^{T}}\left[X C^{T}-B^{C}\right]+\frac{\partial D(x)^{-1}}{\partial x^{T}}[X C-B]\right\} \\
& =\left(C^{T} \otimes D(x)^{-1}\right) \frac{\partial \operatorname{vec}\{X\}}{\partial x^{T}}+\left(C \otimes D(x)^{-T}\right) \frac{\partial \operatorname{vec}\{X\}}{\partial x^{T}} \\
& +\alpha\left[\frac{\partial \operatorname{vec}\{D(x)\}}{\partial x^{T}}\right]^{T} \frac{\partial \operatorname{vec}\left\{D(x)^{-T}\right\}}{\partial x^{T}} \\
& =\left(C^{T} \otimes D(x)^{-1}\right)+\left(C \otimes D(x)^{-T}\right) \\
& -\alpha\left[\frac{\partial \operatorname{vec}\{D(x)\}}{\partial x^{T}}\right]^{T}\left(D(x)^{-1} \otimes D(x)^{-T}\right)\left[\frac{\partial \operatorname{vec}\{D(x)\}}{\partial x^{T}}\right]^{C} .
\end{aligned}
$$

Substitution of the definition (133) for $E(x)$ into this expression then completes the proof.

\section{ACKNOWLEDGMENT}

The authors would like to thank Dr. R. Moheimani and Dr. A. Fleming for providing the cantilevered beam experimental data and for their insights into aspects of its modelling.

\section{REFERENCES}

[1] S. Boyd and L. Vandenberghe, Convex Optimization. Cambridge, U.K.: Cambridge Univ. Press, 2004.

[2] D. R. Brillinger, Time Series: Data Analysis and Theory. San Francisco, CA: Holden-Day, 1981.

[3] T. Cover and J. Thomas, Elements of Information Theory. New York: Wiley, 1991

[4] A. P. Dempster, N. M. Laird, and D. B. Rubin, "Maximum likelihood from incomplete data via the EM algorithm," J. Royal Stat. Soc., ser. B, vol. 39, pp. 1-38, 1977.

[5] J. E. Dennis and R. B. Schnabel, Numerical Methods for Unconstrained Optimization and Nonlinear Equations. Englewood Cliffs, NJ: Prentice Hall, 1983.

[6] S. Gibson and B. Ninness, "Robust maximum-likelihood estimation of multivariable dynamic systems," Automatica, vol. 41, no. 10, pp. 1667-1682, 2005.

[7] S. Gibson, A. Wills, and B. Ninness, "Maximum-likelihood parameter estimation of bilinear systems," IEEE Trans. Automat. Control, vol. 50, no. 10, pp. 1581-1596, Oct. 2005.

[8] H. Hindi, "A tutorial on convex optimization," in Proc. Amer. Control Conf., 2004, pp. 3252-3262.

[9] Horn and Johnson, Matrix Analysis. Cambridge, U.K.: Cambridge University Press, 1985.

[10] A. H. Jazwinski, Stochastic Processes and Filtering Theory. New York: Academic Press, 1970.

[11] J. N. Juang, Applied System Identification. Englewood Cliffs, NJ: Prentice Hall, 1994

[12] L. Ljung, MATLAB System Identification Toolbox Users Guide, 6 ed. New York: The Mathworks, 2004.

[13] J. R. Magnus and H. Neudecker, Matrix Differential Calculus with Applications in Statistics and Econometrics. New York: Wiley, 1988.
[14] J. R. Magnus and H. Neudecker, Matrix Differential Calculus with Applications in Statistics and Econometrics. Chichester, U.K.: Wiley, 1988.

[15] T. McKelvey, "Frequency domain identification," in Proc. SYSID'00, Santa Barbara, CA, 2000.

[16] T. McKelvey, "Frequency domain identification methods," Circuits Syst. Signal Processing, vol. 21, no. 1, pp. 39-55, 2002.

[17] T. McKelvey, H. Akçay, and L. Ljung, "Subspace-based multivariable system identification from frequency response data," IEEE Trans. Automat. Control, vol. 41, no. 7, pp. 960-979, Jul. 1996.

[18] R. Pintelon, P. Guillaume, Y. Rolain, J. Schoukens, and H. Van hamme, "Parametric identification of transfer functions in the frequency domain-A survey," IEEE Trans. Automat. Control, vol. 39, no. 11, pp. 2245-2260, Nov. 1994.

[19] J. Nocedal and S. Wright, Numerical Optimization. New York: Springer-Verlag, 1999.

[20] R. Pintelon and I. Kollar, "On the frequency scaling in continuous-time modeling," IEEE Trans. Instrum. Meas., vol. 54, no. 1, pp. 318-321, Feb. 2005.

[21] R. Pintelon and J. Schoukens, System Identification: A Frequency Domain Approach. Piscataway, NJ: IEEE Press, 2001.

[22] J. Schoukens, G. Vandersteen, R. Pintelon, and P. Guillaume, "Frequency domain identification of linear systems using arbitrary excitations and a nonparametric noise model," IEEE Trans. Automat. Control, vol. 44, no. 2, pp. 343-347, Feb. 1999.

[23] A. Weinmann, Uncertain Models and Robust Control. New York: Springer-Verlag, 1991

[24] A. G. Wills, D. Bates, A. Fleming, B. Ninness, and R. Moheimani, "Application of MPC to an active structure using sampling rates up to 25 kHz," in Proc. CDC/ECC'05, Dec. 2005, pp. 3176-3181.

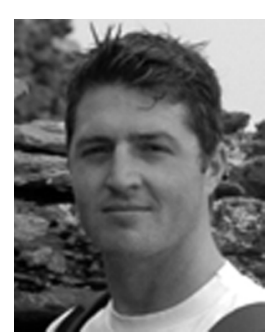

Adrian Wills was born in 1976 in Orange, N.S.W. Australia, in 1976. He received the B.E. and Ph.D. degrees in electrical engineering from The University of Newcastle, Newcastle, Australia (Callaghan Campus) in 1999 and 2003, respectively.

Since then he has held a Postdoctoral Research position at Newcastle University and was awarded an Australian Postdoctoral Fellowship in 2007. The focus of his research has been in the areas of system identification and model predictive control.

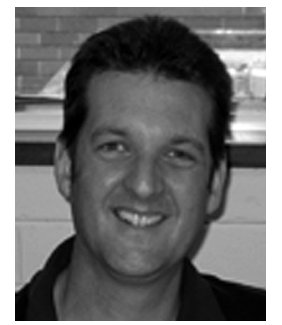

Brett Ninness was born in Singleton, Australia, in 1963. He received the B.E., M.E., and Ph.D. degrees in electrical engineering from the University of Newcastle, Newcastle, Australia, in 1986, 1991, and 1994 respectively.

He has stayed with the School of Electrical Engineering and Computer Science at the University of Newcastle since 1993, where he is currently a Professor. His research interests are in the areas of system identification and stochastic signal processing, in which he has authored approximately 100 papers in journals and conference proceedings.

Dr. Ninness has served on the editorial boards of Automatica, the IEEE TRANSACTIONS ON AUTOMATIC CONTROL, and is currently Editor-in-Chief for IEE Control Theory and Applications. He jointly organized the 14th IFAC Symposium on System Identification in Newcastle, Australia in 2006.

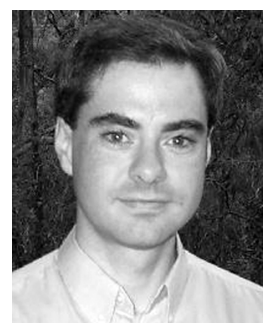

Stuart Gibson received the B.Sc. degree, the B.E. degree in computer engineering, and the Ph.D.degree in electrical engineering from the University of Newcastle, Newcastle, Australia, in 1993, 1996, and 2004 , respectively.

From 1995 to 1997, he was a Physicist in the M.R.I. Unit, John Hunter Hospital, Newcastle, before joining the Centre for Integrated Dynamics and Control (CIDAC) in 1997, where he stayed until 2000 . He is currently working within the financial industry. 